nephron

Experimental

Nephrology

and Genetics
Nephron 2019;142:328-350

DOI: $10.1159 / 000499506$
Received: November 20, 2018

Accepted after revision: March 10, 2019

Published online: May 2, 2019

\title{
Spontaneous Extracellular Matrix Accumulation in a Human in vitro Model of Renal Fibrosis Is Mediated by aV Integrins
}

\author{
Hélène Bon Paul Hales Simon Lumb Gill Holdsworth Tim Johnson \\ Omar Qureshi Breda M. Twomey
}

UCB Pharma, Berkshire, UK

\section{Keywords}

Renal fibrosis - Extracellular matrix - Epithelial cells .

Fibroblasts · Integrin

\begin{abstract}
Background: Tubulointerstitial fibrosis is a key feature of chronic kidney diseases leading to renal failure. It is characterised by the infiltration of fibroblasts and aberrant accumulation of extracellular matrix (ECM) proteins, which are associated with progressive loss of renal function. Integrins play a major role in fibrosis, but the mechanisms through which they do this are not fully understood. Objective: Using a complex cell system, we test the hypothesis that integrins are pro-fibrotic via regulation of functional interactions between tubular epithelial cells and renal fibroblasts. Method: Contact co-culture of human primary renal proximal tubular epithelial cells and renal fibroblasts promoted the spontaneous accumulation of a mature ECM rich in interstitial collagens, which was considerably in excess of that seen in the individual mono-cultures. Both cell types persisted through-
\end{abstract}

\begin{tabular}{ll}
\hline KARGER & ( 2019 The Author(s) \\
Published by S. Karger AG, Basel & Karger \\
E-Mail karger@karger.com & $\begin{array}{l}\text { This article is licensed under the Creative Commons Attribution- } \\
\text { NonCommercial-NoDerivatives 4.0 International License (CC BY- } \\
\text { www.karger.com/nef }\end{array}$ \\
$\begin{array}{l}\text { NC-ND) (http://www.karger.com/Services/OpenAccessLicense). } \\
\text { Usage and distribution for commercial purposes as well as any dis- } \\
\text { tribution of modified material requires written permission. }\end{array}$
\end{tabular}

out the culture and were capable of expressing multiple ECM components. Results: While ECM accumulation was inhibited by the clinically proven anti-fibrotic, nintedanib, and was partially abrogated by transforming growth factor $\beta$ neutralisation, its levels did not return to basal, indicating additional pathways were implicated in the pro-ECM response. Application of anti-integrin blocking antibodies and small molecules demonstrated a major role of the $\mathrm{aV}$ integrins in the ECM accumulation during fibroblast: epithelial cell interactions. Conclusion: Integrin-mediated pathways can facilitate the spontaneous accumulation of ECM during fibroblast: epithelial cell interactions, and this direct renal co-culture assay system could provide a translational in vitro assay for investigating novel pathways involved in the pro-ECM response and the screening of renal anti-fibrotic agents.

(c) 2019 The Author(s)

Published by S. Karger AG, Basel

O.Q. and B.T. are joint senior authors. H.B.: Currently at Genmab. O.Q.: Currently at Celentyx.
Dr. Breda M. Twomey

UCB Pharma

208 Bath Road

Slough, Berkshire SL13WE (UK)

E-Mail breda.twomey@ucb.com 


\section{Introduction}

Chronic kidney disease (CKD) affects $8-16 \%$ of adults worldwide and the prevalence rises to almost 50\% after the age of 70 years [1]. There are $>2$ million people worldwide on renal replacement therapy, representing approximately $10 \%$ of those that require it. For those receiving dialysis, then average survival time is $<6$ years and typically provides a low quality of life. CKD therefore represents a huge unmet clinical need.

Loss of organ function in CKD is driven by a progressive fibrosis, with the involvement of the tubulointerstitium associated with the highest risk of end-stage disease. Tubulointerstitial fibrosis is characterised by expansion of the tubular basement membrane due to the excessive accumulation of extracellular matrix (ECM) components and a persistent infiltration of fibroblasts and myofibroblasts. Eventually this fibrotic remodelling becomes selfdriving, irrespective of the primary disease. There have been many attempts to develop therapeutics to treat renal fibrosis; however, there are currently no licensed effective therapies to treat kidney fibrosis and most other types of organ fibrosis [2].

Fibrotic remodelling is initiated by repeated kidney injury such as that due to hypertension, hyperglyceamia or infection causing tubular and glomerular endothelial and epithelial cell stress. This stimulates the infiltration of immune cells such as macrophages, which release soluble factors in the damaged tissue including transforming growth factor $\beta 1$ (TGF- $\beta 1$ ), platelet-derived growth factor (PDGF) and interleukin 4 [3], which ultimately leads to the proliferation and activation of fibroblasts into myofibroblasts that deposit ECM into the surrounding connective tissue. Fibroblast accumulation in the context of fibrosis has been widely described [4]. Studies have also shown the differentiation of tubular epithelial cells into a more mesenchymal fibroblast-like phenotype, which in some studies is referred to as epithelial mesenchymal transition, facilitating the epithelial cells to become major producers of ECM [5]. In healthy organs, the proteins of the mature ECM are maintained in a homeostatic balance between synthesis, deposition and proteolytic clearance. In contrast, in fibrotic disease, the rate of matrix protein synthesis and deposition outweighs breakdown, leading to excessive ECM accumulation. Irrespective of the source, this large build-up of ECM proteins leads to tubular basement membrane, glomerular basement membrane and mesangial matrix expansion so is a primary driver of disease.

Although much of the pathogenesis of renal fibrosis remains to be elucidated, the excessive accumulation of ECM is a defining feature of the disease, and fibroblasts and tubular epithelial cells have emerged as the major effector cells. Subsequently, ECM quantitation and characterization offer the best assessment of disease progression and thus therapeuticintervention efficacy. To this end, we have recently developed a series of high-throughput cell generated assays in 384 well format using either radioisotopic incorporation, immunofluorescent staining or total protein staining of ECM proteins as fibrotic endpoints $[6,7]$. In the development of these approaches, there was an opportunity to try and recreate an in vivo-like system by using combinations of cells rather than mono-cultures. Earlier studies have suggested crosstalk occurred between various cell types $[8,9]$ although the full effect of this on the mature ECM has never been established. Our initial studies combining renal proximal tubular epithelial cell (RPTEC) and human renal fibroblasts (HRF) in a direct co-culture clearly led to increased levels of mature ECM without any additional stimulation, suggesting that those cellular interactions were crucial to trigger the accumulation of matrix [7].

The central role of integrins in mediating the pathogenesis of fibrosis has been demonstrated in many in vivo studies using a combination of genetic and pharmacological approaches. Myofibroblast-specific deletion of $\alpha \mathrm{V}$ in mouse models of liver, kidney and lung fibrosis was shown to have a significant anti-fibrotic effects; this inhibitory effect was replicated with a small molecule $\alpha \mathrm{V}$ inhibitor in lung and kidney models of fibrosis and a reversal of liver fibrosis [10]. Dual blockade of the integrins, $\alpha \mathrm{V} \beta 3$ and $\alpha \mathrm{V} \beta 5$, with the small molecule inhibitor, cilengitide prevented systemic fibrosis in a murine model of sytemic sclerosis [11]. Kidney fibrosis after unilateral ureteric obstruction was protected by a8 deletion [12] but exacerbated by proximal tubular E-cadherin deficiency, which was demonstrated to be mediated by a 3 integrindependent signalling [13]. While these in vivo studies highlight an important role for integrins in the modulation of fibrosis in mouse models, translatability to human fibrotic disease has yet to be demonstrated.

There are a number of integrin molecules that have progressed to clinical trials, such as abituzumab, a human $\alpha \mathrm{V}$-specific antibody initially shown to block TGF$\beta$-induced myofibroblast activation in vitro [14] which was in a Phase II trial for scleroderma but is now terminated due to enrollment difficulties. The anti-integrin aV $\beta 6$ antibody from Biogen, BG00011 (formally STX100 ) and the small molecule $\alpha \mathrm{V} \beta 6$ inhibitor from GSK (GSK3008348) are both undergoing trials in idiopathic pulmonary fibrosis (IPF) [15]. In kidney fibrosis, a clinical trial has been completed with the $\alpha 5 \beta 3$ antibody 
from Janssen/Vascular Pharmaceutical Inc. (VPI-2690B) in diabetic nephropathy, but the trial was reported not to meet its primary end-point. Even given the large amount of activity in the area of integrins in fibrosis, the precise mechanism of action of anti-integrin therapeutics remains unclear. This work describes a human in vitro model system that may be more predictive to human renal fibrotic disease and could facilitate the testing of multiple anti-fibrotic inhibitors targeted at integrins and a broader range of potential targets.

In this study, we proposed the hypothesis that integrins are pro-fibrotic by regulating functional interactions between epithelial cells and fibroblasts, and possibly also between these cell types and the extracellular environment. To test this hypothesis, we exploited our previously described contact co-culture of RPTECs and HRF with the resultant accumulation of ECM as a model system to explore the role of integrins and more specifically the $\alpha \mathrm{V}$ integrin sub-family. We demonstrated a central contribution of $a \mathrm{~V}$ integrins in the contact-driven, ECM deposition using well-described blocking anti-integrin $\alpha \mathrm{V}$ antibodies. In addition, inhibitory effects were shown with a number of previously described small molecule anti-fibrotic inhibitors [16], showing a bias towards those molecules targeting integrin-cell interactions.

\section{Materials and Methods}

\section{Cell Culture and Treatment}

Human primary RPTECs were purchased from Innoprot (P10662) and were maintained in Renal Epithelial Cell Basal Medium (ATCC PCS-400-030) supplemented with Renal Epithelial Cell Growth Kit Components (ATCC PCS-400-040). HRF were purchased from Innoprot (P10666) and maintained in DMEM F12 (Invitrogen) containing 10\% FCS and supplemented with $2 \mathrm{mM}$ L-glutamine. For experiments, epithelial cell medium was used for both cell types. Cells were grown in $100 \%$ humidity and $5 \% \mathrm{CO}_{2}$ at $37^{\circ} \mathrm{C}$.

For antibody blockade, cells were incubated in the presence of anti-TGF $\beta$ (MAB1835, R\&D), anti-integrin $\alpha \mathrm{V}$ (MABT207, Millipore), anti-integrin $\alpha \mathrm{V} \beta 5$ (MAB2528, R\&D) and anti-integrin $\alpha \mathrm{V} \beta 6$ (ab77906, Abcam), at the indicated concentrations for the duration of the culture period. Appropriate isotype controls were included.

For treatment with small molecule inhibitors, cells were incubated in the presence of nintedanib (Selleckchem), pirfenidone (Sigma-Aldrich), bindarit (Abcam), RS504393 (Sigma-Aldrich), beraprost sodium (Tocris Bioscience), IVA337 (UCB), obeticholic acid (Selleckchem), sarpogrelate hydrochloride (Sigma-Aldrich), TRAM34 (Sigma-Aldrich), nilotinib (Abcam), baricitinib (Selleckchem), rapamycin (Sigma-Aldrich), FTY720 (Sigma-Aldrich), pyridoxamine dihydrochloride (Sigma-Aldrich), BML-111 (Abcam), L3223 (Echelon Biosciences), enalapril maleate (Sigma-Al- drich), emricasam (Selleckchem), CWHM12 (UCB), C8 (UCB), Cyclo (RGDyK) (Selleckchem), Cyclo (RGDfK) (Selleckchem), SB273005 (Selleckchem), cilengitide (Selleckchem) and DC9711 (DC Chemicals) at the indicated concentrations. Compounds prepared as DMSO stock solutions were diluted in medium prior to addition to cells and were included at the indicated concentrations for the duration of the culture period. DMSO vehicle control was included in these experiments.

\section{Measurement of Cell Growth}

Cell growth was assessed using PrestoBlue ${ }^{\circledR}$ Cell Viability reagent (Thermo Fischer Scientific) following the instructions of the manufacturer.

\section{Flow Cytometry}

Cells were detached using Accutase and labelled as described for immunofluorescence with AlexaFluor488-conjugated anti-cytokeratin 18 (eBioscience, LDK18, 53-9815-82), FITC-conjugated anti-CD90/Thy1 (eBioscience, 11-0909) and anti- $\alpha$-Smooth muscle actin (Abcam) antibodies. For integrin staining, live cells were stained using anti-integrin $\alpha \mathrm{V}$ (Millipore), anti-integrin $\beta 1$ (R\&D), anti-integrin $\beta 5(R \& D)$, anti-integrin $\beta 6(R \& D)$ and anti-integrin $\beta 8(\mathrm{R} \& \mathrm{D})$ antibodies. Cells were then analysed using a BD FACSCanto II. Histograms were prepared using FlowJo (LLC, OR).

\section{Brightfield Images}

Brightfield images of cells in culture were taken with an EVOS XL Core Imaging System (Thermo Fischer Scientific) using a 10× objective.

\section{Immunofluorescence of Cells and ECM}

Cells were plated in dark-walled imaging plates (BD Biosciences or Greiner) or transwell inserts. After 7 days incubation at $37^{\circ} \mathrm{C} 5 \% \mathrm{CO}_{2}$, cells were fixed with $4 \%$ formalin (Sigma-Aldrich) and permeabilised with $0.1 \%$ Triton $\mathrm{X}-100$ for intracellular markers, before being stained using Alexa Fluor488-conjugated cytokeratin 18 (eBioscience, clone LDK18, 53-9815-82), anti-CD90/ Thy1 (eBioscience, 11-0909), anti- $\alpha$-smooth muscle actin (Abcam, ab5694), anti-fibronectin (eBioscience, 14-9869-82), anti-collagen I (Millipore, AB745), anti-collagen III (Millipore, AB747), anticollagen IV (eBioscience, 14-9871-82), anti-collagen V (Abcam, ab7046), anti-collagen VI (Abcam, ab6588), anti-Integrin Alpha V (Millipore, MABT207), anti-Integrin Beta 1 (R\&D, MAB1778), anti-Integrin Beta $5(\mathrm{R} \& \mathrm{D}, \mathrm{AB} 3825)$, anti-Integrin Beta $6(\mathrm{R} \& \mathrm{D}$, MAB4155), anti-Integrin Beta 8 (R\&D, MAB4775) antibodies and AlexaFluor-labelled secondary antibodies (Life Technologies). Cells were stained using DAPI (Thermo Fisher, D3571) and were scanned on the Arrayscan HC reader (Cellomics) using a 2-channel protocol under the "Cellomics CellHealth Profiling" bioapplication and a $20 \times$ objective (X1 camera) with $2 \times 2$ binning $(1104 \times$ 1104 pixels/field).

For the measurement of individual ECM components using immunofluorescence, cells were plated in dark-walled imaging plates (BD Bioscience or Greiner, 781090) or Corning transwell plates (3392). After 7 days incubation at $37^{\circ} \mathrm{C} 5 \% \mathrm{CO}_{2}$, cells were washed in PBS and lysed with $20 \mu \mathrm{L} 0.25 \mathrm{M} \mathrm{NH}_{4} \mathrm{OH}$ in $25 \mathrm{mM}$ Tris (Sigma-Aldrich) for $15 \mathrm{~min}$ at $37^{\circ} \mathrm{C}$. Matrix was then washed 3 times in PBS, fixed in $40 \mu \mathrm{L} 100 \%$ methanol for $30 \mathrm{~min}$ at $20^{\circ} \mathrm{C}$ and washed 3 times in PBS before being stained using Alexa Fluor488-conjugated anti-Fibronectin (eBioscience, clone FN-3, 53- 
9869-82), anti-collagens I and III (Millipore, AB745 and AB747), anti-collagen IV (eBioscience, clone 1042, 14-9871-82), anti-collagen V (Abcam, ab7046) and anti-collagen VI (Abcam, ab6588) antibodies. Anti-collagens I and III and IV were detected using either Alexa Fluor555-conjugated or AlexaFluor647 secondary antibodies (Life Technologies). Plates were scanned on the Arrayscan HC reader (Cellomics) using a 4-channel protocol under the "Cellomics CellHealth" profiling bioapplication and a $10 \times$ objective (X1 camera) with $2 \times 2$ binning $(1104 \times 1104$ pixels/field $)$.

\section{Total ECM Measurement}

Immunofluorescence plates were washed 3 times in Flowfusor water before being stained using Flamingo ${ }^{\mathrm{TM}}$ fluorescent gel stain reagent (BioRad) as described previously (Holdsworth et al. [6]). Plates were scanned on the Arrayscan HC reader (Cellomics) using a 2-channel protocol under the "Cellomics CellHealth" profiling bioapplication and a $10 \times$ objective (X1 camera) with $2 \times 2$ binning $(1104 \times 1104$ pixels/field $)$.

\section{Colour-Coding}

Cells were stained with CellTrace ${ }^{\mathrm{TM}}$ Violet or CellTrace ${ }^{\mathrm{TM}}$ Yellow (Invitrogen) following the instructions of the manufacturer.

\section{Affymetrix Bead Array Data}

RNA extraction and microarray analyses were performed by Almac Diagnostics, Craigavon. In brief, RNA was extracted from snap-frozen cells following the RNA-Stat 60 solvent extraction protocol. Further purification and elution were carried out using RNeasy MiniElute spin columns. Purified RNA was amplified using the $\mathrm{NuGEN}^{\mathrm{TM}}$ Ovation $^{\mathrm{TM}}$ RNA Amplification System V2, cDNA was subsequently fragmented and labelled with the Encore Biotin Module. Hybridisations were carried out using Affymetrix GeneChip ${ }^{\circledR}$ Human Genome U133 Plus 2.0 arrays for $18 \mathrm{~h}$ at $45^{\circ} \mathrm{C}$, washed, stained and scanned with a GeneChip ${ }^{\circledR}$ Scanner 3000.

Raw data (Cel) files were processed with Genedata Expressionist $^{\circledR}$ 9.1 Refiner Array using Robust Multi-Array Average subtraction and quantile normalization. Analysis of data was then performed using Genedata Analyst. T tests were performed between the co-culture and mono-culture chips (using a bootstrap with 10 repeats and the group means for effect size) to generate the Benjamini-Hochberg $q$ value for each probe set. Heat maps were generated for panels of probe sets by hierarchical clustering, using the positive correlation (1-r) model to calculate distance between the probe sets, with complete linkage. Individual probe sets were selected to demonstrate expression of particular genes by first filtering so that a signal of at least 30 was observed on at least one of the chips, then by Affymetrix name suffix (i.e., _at > _a_at $>$ _s_at $>$ _x_at), and finally where multiple probe sets existed by the lowest $q$ value generated by the comparisons between epithelial cells, fibroblasts or a grouping of all mono-cultures with the co-culture chips.

\section{Results}

Contact Co-Culture of Epithelial Cells and Fibroblasts Results in Spontaneous Accumulation of ECM

Commercially available human primary RPTEC and primary HRF were characterised by flow cytometry to verify the cells were as close to their primary phenotype as possible. A primary culture of RPTEC that showed high expression of cytokeratin 18 and was CD90/Thy1 negative (online suppl. Fig. S1a; for all online suppl. material, see www.karger.com/doi/10.1159/000499506) and a primary culture of HRF that was negative for cytokeratin 18 and showed strong expression of CD90/Thy1 (online suppl. Fig. S1b) were selected for the rest of the study.

Following the co-culture of both cell types in a 1:1 ratio in vitro for 7 days, cells were removed using ammonium hydroxide to leave the intact mature deposited ECM on the plate. The ECM was then immunostained for fibronectin, collagens I, III, IV, and V and later re-stained with a fluorescent total protein stain (Flamingo ${ }^{\mathrm{TM}}$ Fluorescent Gel Stain [6]). Resulting images were captured using a high-content fluorescent imaging platform so both individual and total ECM component proteins could be visualised (online suppl. Fig. S2).

Consistent with our previous publication [7], the coculture of fibroblasts and epithelial cells led to the spontaneous enhanced accumulation of total ECM compared to the mono-cultures alone. Significant increases in the individual matrix components fibronectin, collagens I, III and V were also observed. In contrast, mature collagen IV was clearly visible in the epithelial mono-culture ECM, not detectable in fibroblast ECM and typically less in the co-culture although the intensity of signal obtained for this marker was considerably lower, thus more variable, than for the other ECM proteins (Fig. 1).

To test the importance of direct cell interactions in mediating the pro-fibrotic phenotype in the co-culture, a transwell experiment was first conducted in which each cell type was grown in spatially separated compartments, in a well with either the same or the other cell type in the transwell above. This allows the circulation of soluble factors but prevents direct cell contact. The presence of fibroblasts in the upper transwell did not significantly enhance the amount of matrix accumulated by the epithelial cells present in the lower transwell compared with epithelial cells in both compartments (Fig. 2a). Similarly, the presence of epithelial cells in the upper transwell did not increase the matrix laid down by the fibroblasts in the lower transwell, relative to fibroblasts top and bottom (Fig. 2b). It should also be noted that the ECM signal is very low in both the lower compartments whether there is epithelial cells or fibroblasts present and this signal was not changed significantly in the co-culture format. This result indicated that direct contact between both cell types was most likely required for enhanced ECM generation but does not definitely exclude the possibility that 
Total ECM proteins

Flamingo
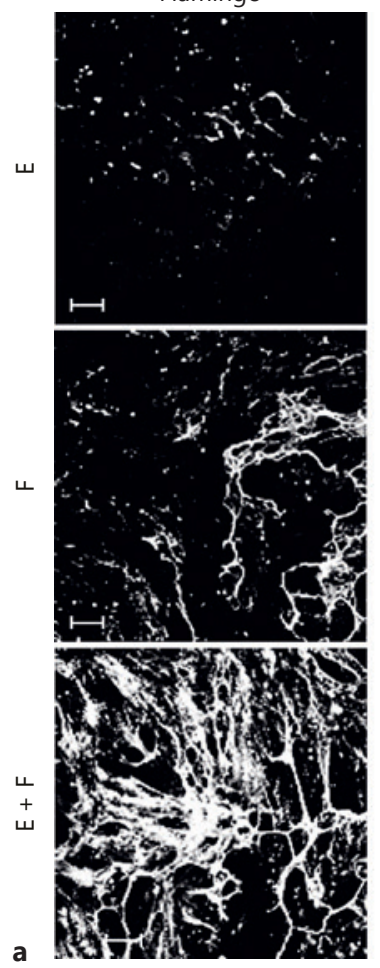

Collagens | \& III
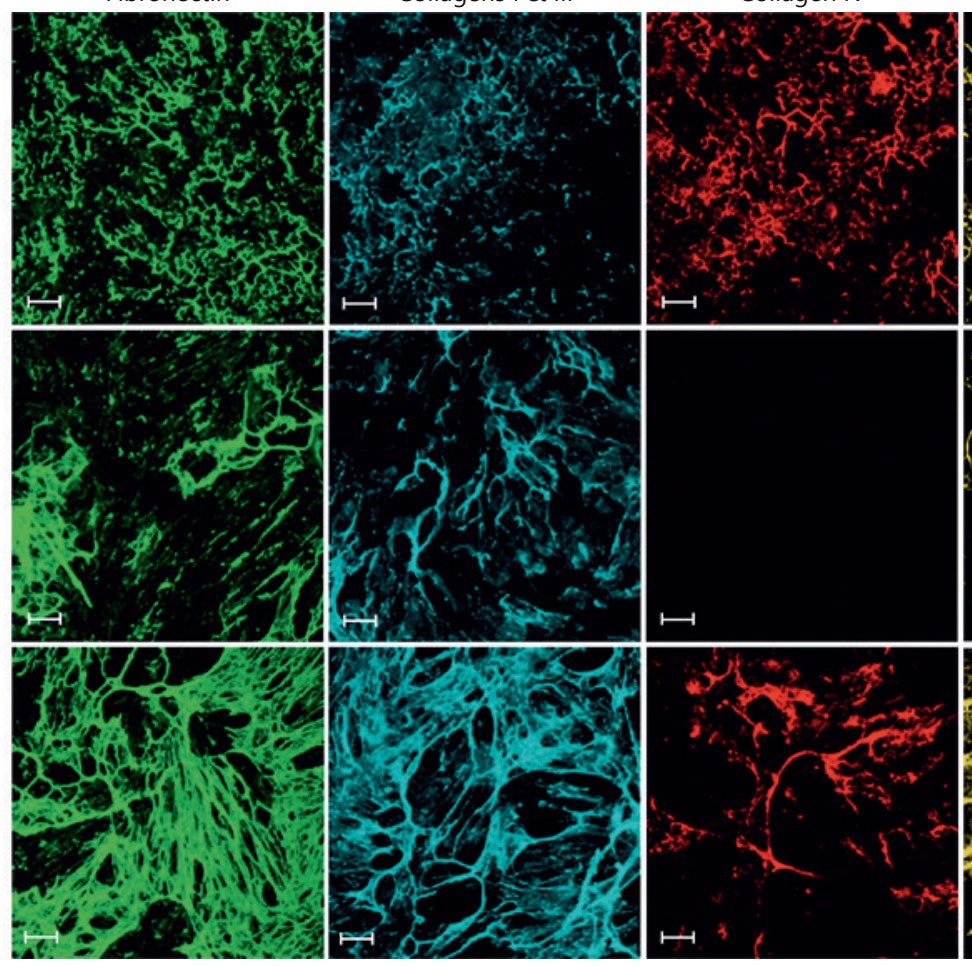

Collagen V

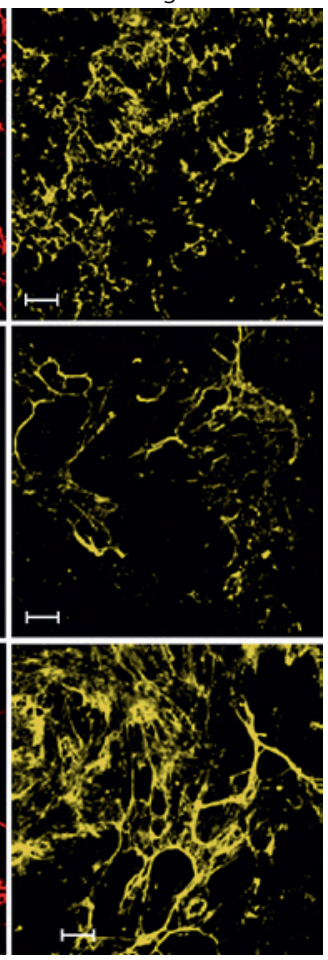

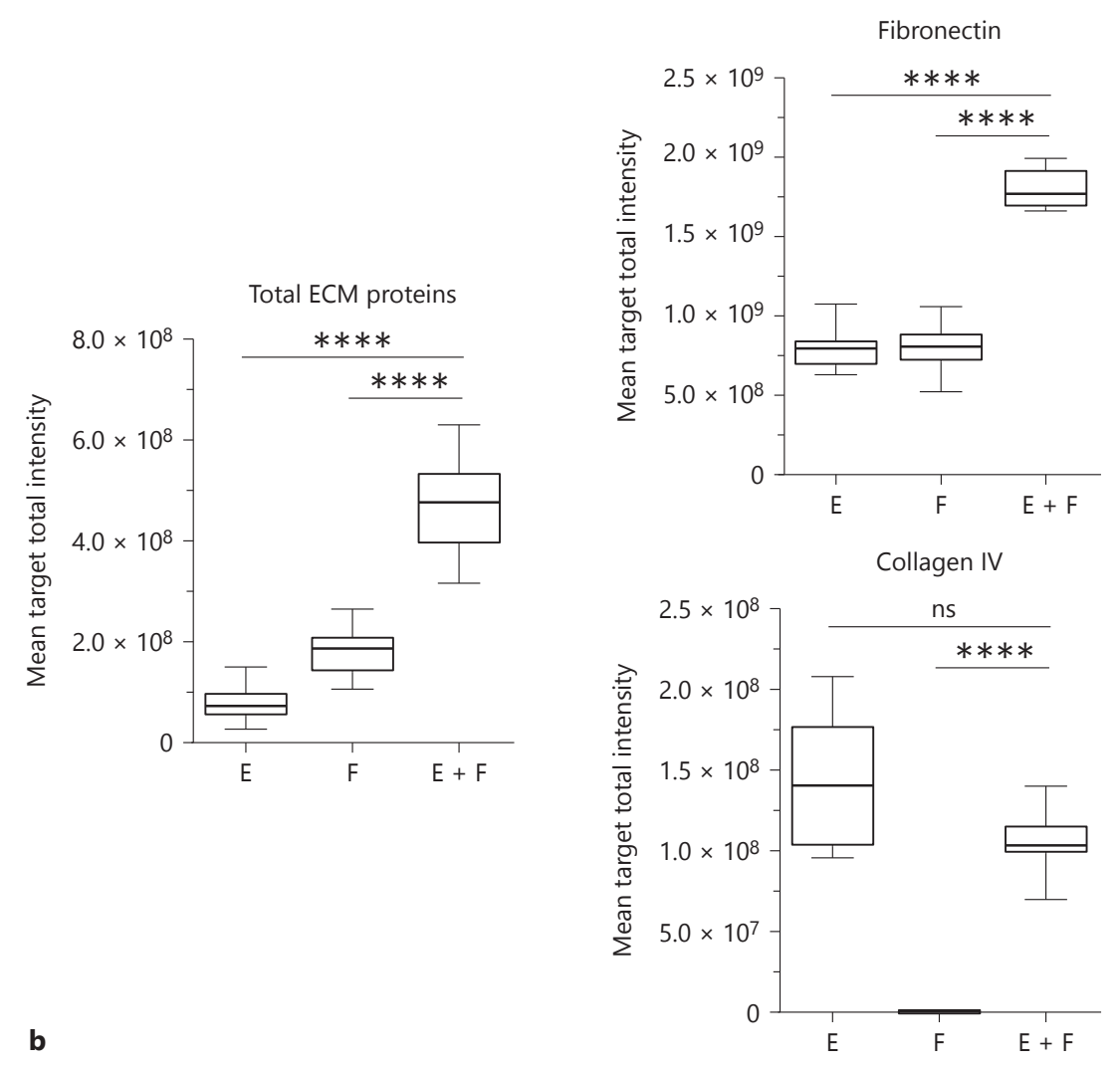

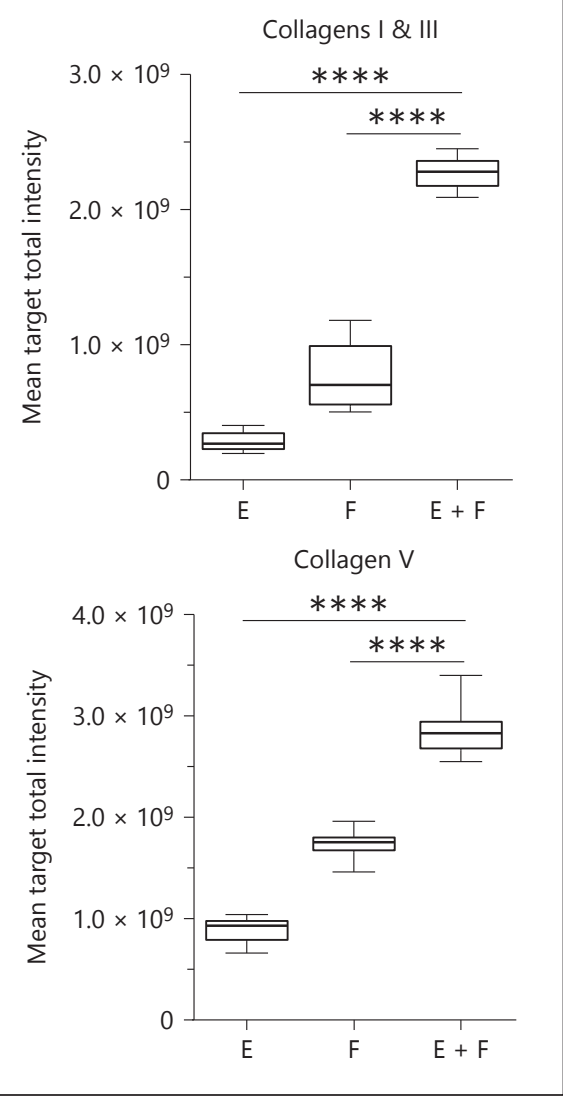

(For legend see next page.)

Bon/Hales/Lumb/Holdsworth/Johnson/ Qureshi/Twomey 
soluble factors exchanged between cell types might contribute even though we were unable to detect in our model system.

\section{Both Epithelial Cells and Fibroblasts Contribute to ECM Production in Contact Co-Culture}

To further understand the underlying mechanisms of ECM accumulation, we monitored the accumulation of the ECM in the co-culture over time. Increases in ECM were observed as early as day 3 and were dominated by fibronectin. Increased accumulation of collagens was observed more clearly at later time points from day 5 onwards (Fig. 3a). Immunofluorescence images showed significant co-localisation of the collagen and fibronectin staining (Fig. 3b). These results are consistent with a model whereby fibronectin is laid down as an initial scaffold for collagen assembly [17].

To begin to understand the contribution of each cell type, individual cells were colour coded using fluorescent chemical dyes. Epithelial cells were labelled with Cell Trace ${ }^{\mathrm{TM}}$ Violet (blue) and the fibroblasts with Cell Trace $^{\mathrm{TM}}$ Yellow (orange). Both mono- or co-cultures showed good retention of the 2 dyes over 7 days (online suppl. Fig. S3). Different ratios of epithelial cells to fibroblasts were then titrated in the co-culture system to establish whether the changes in ECM were in part due to one dominating cell type. We observed that even small numbers of epithelial cells co-cultured with fibroblasts, or small numbers of fibroblasts co-cultured with epithelial cells, led to increases in matrix accumulation, indicating that either cell type was capable of contributing. However, the increase was maximal at 1:1 cell ratio for total ECM proteins, fibronectin and collagens I and III, showing 2.3-, 3- and 1.8-fold increased signal, respectively, compared to mono-culture controls. Fibronectin was the most highly up-regulated ECM protein in the contact co-culture, in contrast to collagen IV which could be accumulated at low levels by mono-cultures of epithelial cells, and collagen $\mathrm{V}$ by mono-cultures of fibroblasts (Fig. 4a). Quantification of the numbers of each cell type present at day 1 and 7 across the different cell

Fig. 1. Co-culture of epithelial cells and fibroblasts leads to spontaneous ECM production. Epithelial cells and fibroblasts were cultured for 7 days in mono- or co-culture (1:1 ratio). Cells were then lysed; ECM was fixed and stained using a total protein dye or specific antibodies. a Images of total ECM components (total ECM proteins, white;), and individual ECM components (fibronectin, green; collagens I and III, blue; collagen IV, red; collagen V) are representative of 4 independent experiments. b Graphs show the ratios showed that the ratio of cells remained consistent throughout the culture, suggesting that one cell type did not appear to dominate over the other so both epithelial cells and fibroblasts could make a contribution to the final ECM accumulation (Fig. 4b).

To test this finding, intracellular expression of ECM proteins was examined in the colour coded cells in coculture at the optimal 1:1 ratio. This allowed ECM expression to be individually associated with each cell type (Fig. 5a). Fibronectin, collagens IV, V and VI were either low or undetectable in both epithelial cells and fibroblasts at the start of the culture (day 1) but showed a significant increase at day 7. In contrast, collagens I and III were already expressed intracellularly by both cell types and demonstrated a more modest increase at day 7 of culture. Collagen V and VI expression was greatly enhanced after 7 days of culture in the fibroblasts only, while fibronectin expression was dramatically induced in both epithelial cells and fibroblasts. This was further exemplified by showing the increased cell-associated fibronectin in the co-culture compared to the respective mono-cultures going from day 1 to 7 (online suppl. Fig. S4). This suggested that the direct contact of the epithelial cells with the fibroblasts triggered fibronectin production, followed by its secretion into the ECM.

Using gene microarray, the early expression of major ECM genes at $48 \mathrm{~h}$ in both the mono-culture of epithelial cells or fibroblasts and in the co-culture was investigated. Transcripts encoding fibronectin and various collagens were expressed by both the epithelial cells and fibroblasts, which demonstrated that both cell types could be producers of the mature matrix components (Fig. 5b; Table 1). Surprisingly, however, in the co-culture system, there was no significant increase in the gene expression profile of the matrix components compared to the mono-cultures, implying that the control of up-regulation was exercised at the level of protein expression, ECM assembly or clearance. This would be consistent with the observation that intracellular changes that were observed in ECM components (Fig. 5a) did not always correlate strictly with the ECM that accumulated (Fig. 1).

results obtained after the quantification of the fluorescent signal from the images. The box and whisker plots show one representative out of 4 independent experiments with 12 replicate wells per condition. E, mono-culture of epithelial cells; F, mono-culture of fibroblasts; E + F, co-culture of epithelial cells and fibroblasts. Images show a single field. Scale bars represent $100 \mu \mathrm{m} .{ }^{*} p<0.05$, ** $p<0.01,{ }^{* * *} p<0.001,{ }^{* * * *} p<0.0001$, one-way ANOVA vs. co-culture. ns, not significant; ECM, extracellular matrix. 


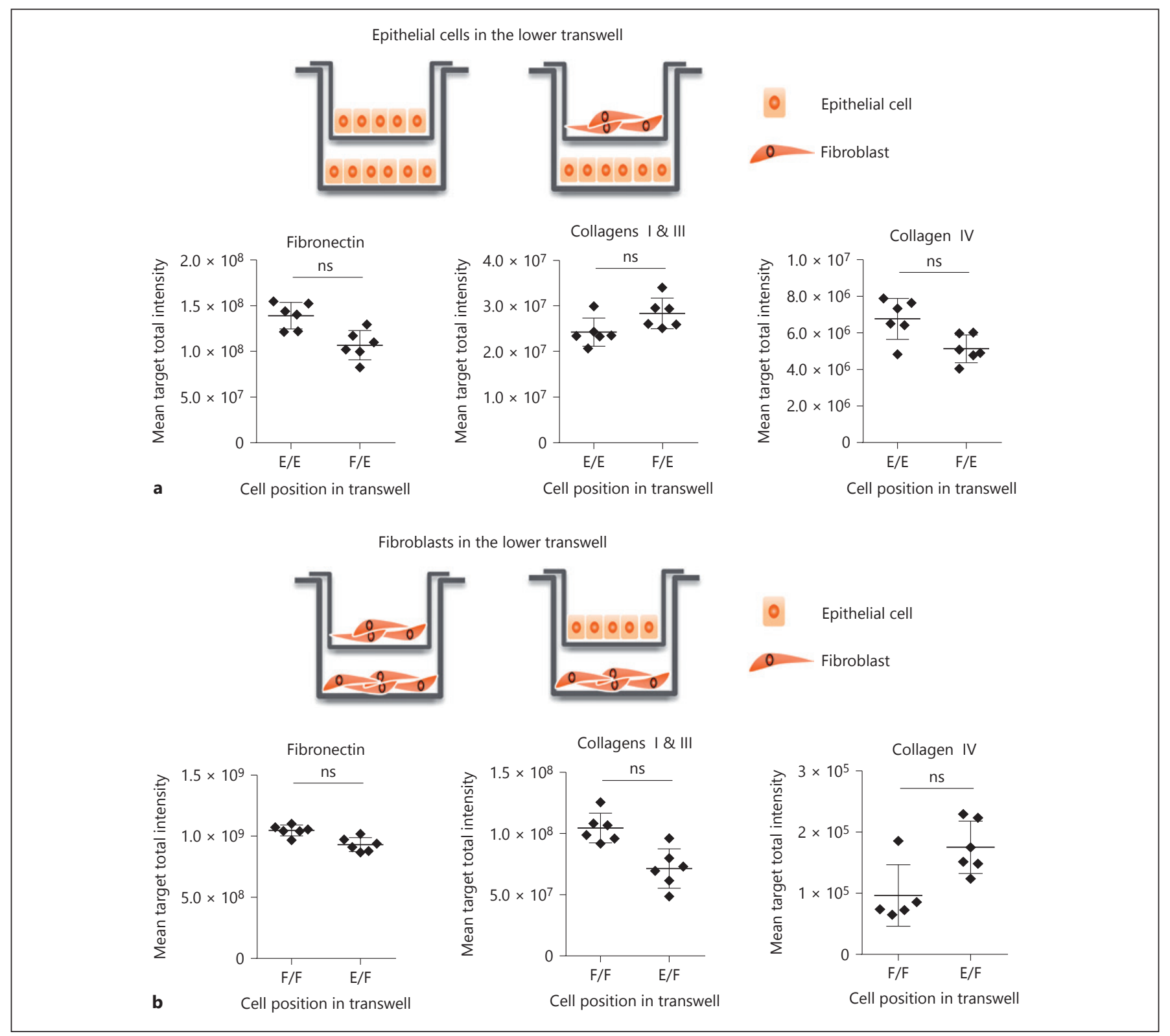

Fig. 2. The direct interaction between epithelial cells and fibroblasts drives the formation of a fibrillary ECM. a Epithelial cells were cultured in the lower well of a transwell system, with either epithelial cells or fibroblasts in the upper well. b Inversely, fibroblasts were cultured in the lower well of a transwell system, with either fibroblasts or epithelial cells in the upper well. After 7 days, cells in the lower well were lysed, and the accumulated ECM was

Fig. 3. Fibronectin, collagens and other ECM components accumulate over time and co-localise in the matrix. a Epithelial cells and fibroblasts were cultured for 9 days in a $1: 1$ ratio and the amount of ECM accumulated over time (at Days 1, 3, 5, 7, and 9) was evaluated. The box and whisker plots show one representative experiment with 6 replicate wells per condition. b Images show the fixed and stained using specific antibodies. Data show mean \pm SD from a representative of 4 independent experiments with 6 replicate wells per condition. E, mono-culture of epithelial cells; F, mono-culture of fibroblasts, $\mathrm{E}+\mathrm{F}$, co-culture of epithelial cells and fibroblasts. ${ }^{*} p<0.05,{ }^{* *} p<0.01,{ }^{* * *} p<0.001,{ }^{* * * *} p<0.0001$, Student $t$ test. ns, not significant.

co-localisation of the total ECM components (total ECM proteins, white) with fibronectin (green) and various collagens (collagens I and III, blue; collagen IV, red; collagen V, yellow; collagen VI, purple) after 7 days in co-culture. Images show a single field. Scale bars represent $100 \mu \mathrm{m}$. ECM, extracellular matrix.

(For figure see next page.) 


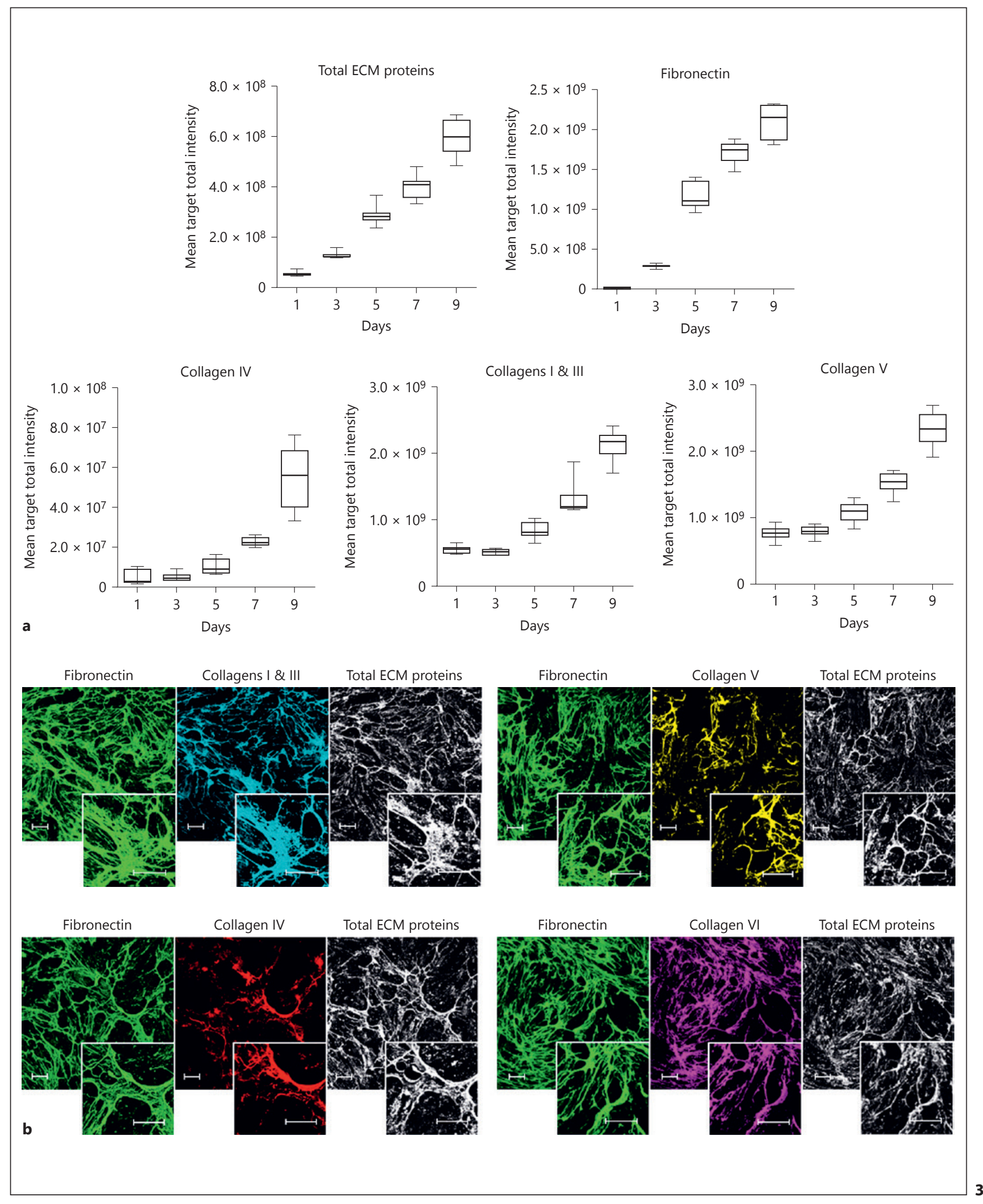

Role of Integrins in a Human in vitro

Nephron 2019;142:328-350 Model of Renal Fibrosis DOI: $10.1159 / 000499506$ 


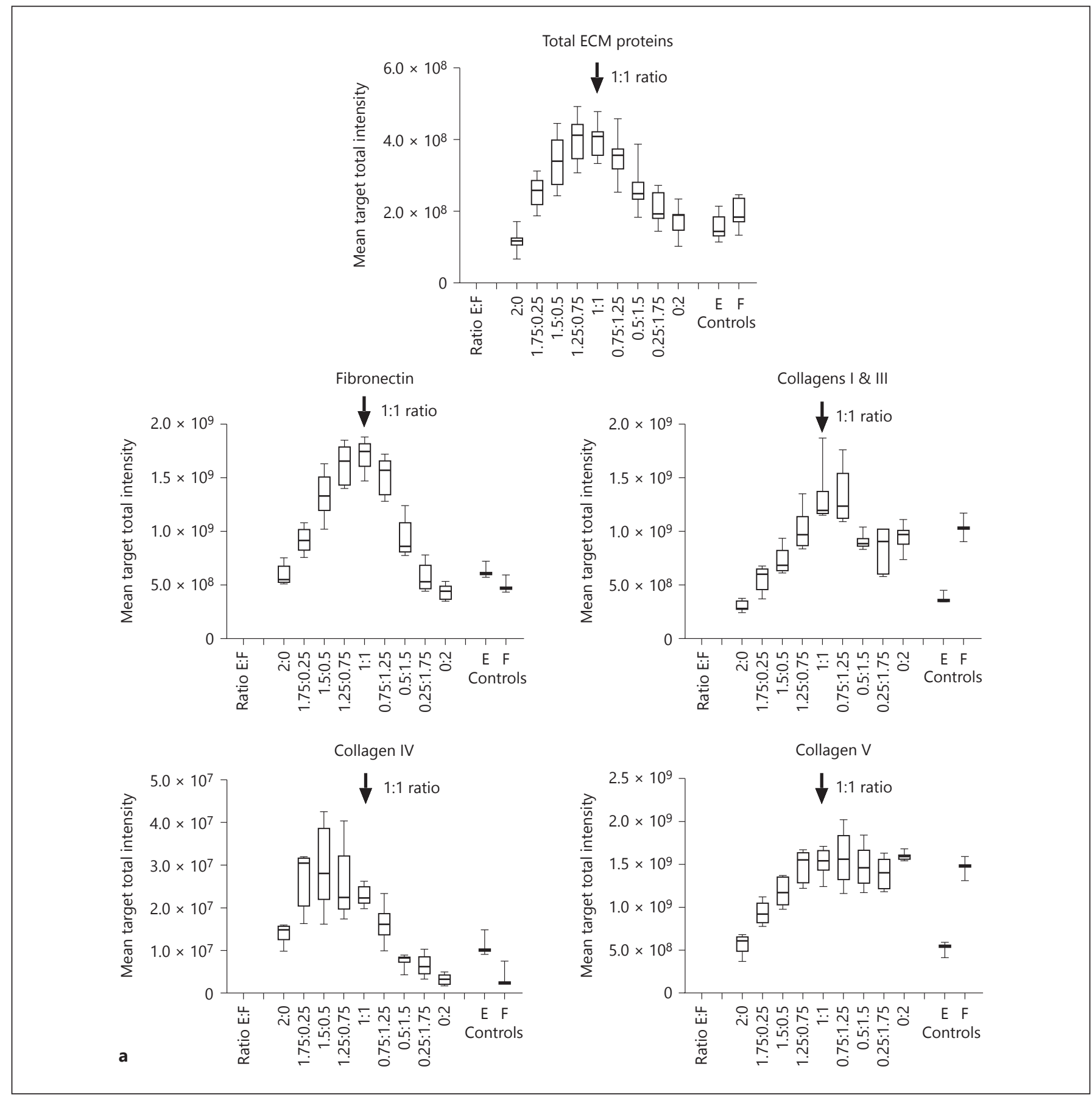

Fig. 4. An equal ratio of epithelial cells and fibroblasts leads to maximum levels of ECM accumulation during contact co-culture. a The amount of ECM (total ECM proteins, fibronectin, collagens I and III, collagens IV and V) accumulated following 7 days in culture after different ratios of colour-coded cells were introduced (Ratio E:F) was measured after the cells were lysed. The signal obtained with non-colour coded cells (Controls) is also shown. Arrows indicate the cell ratio 1:1. The box and whisker plots show a representative of 2 independent experiments with 6 replicate wells per condition. b The corresponding number of epithelial cells (blue) and fibroblasts (orange) was evaluated following 1 or 7 days for the different cell ratios (Ratio E:F) and represented as a percentage of the total cell population at day 1 and 7 . E, mono-culture of epithelial cells; F, mono-culture of fibroblasts; E + F, co-culture of epithelial cells and fibroblasts. ECM, extracellular matrix.

(Figure continued on next page.) 


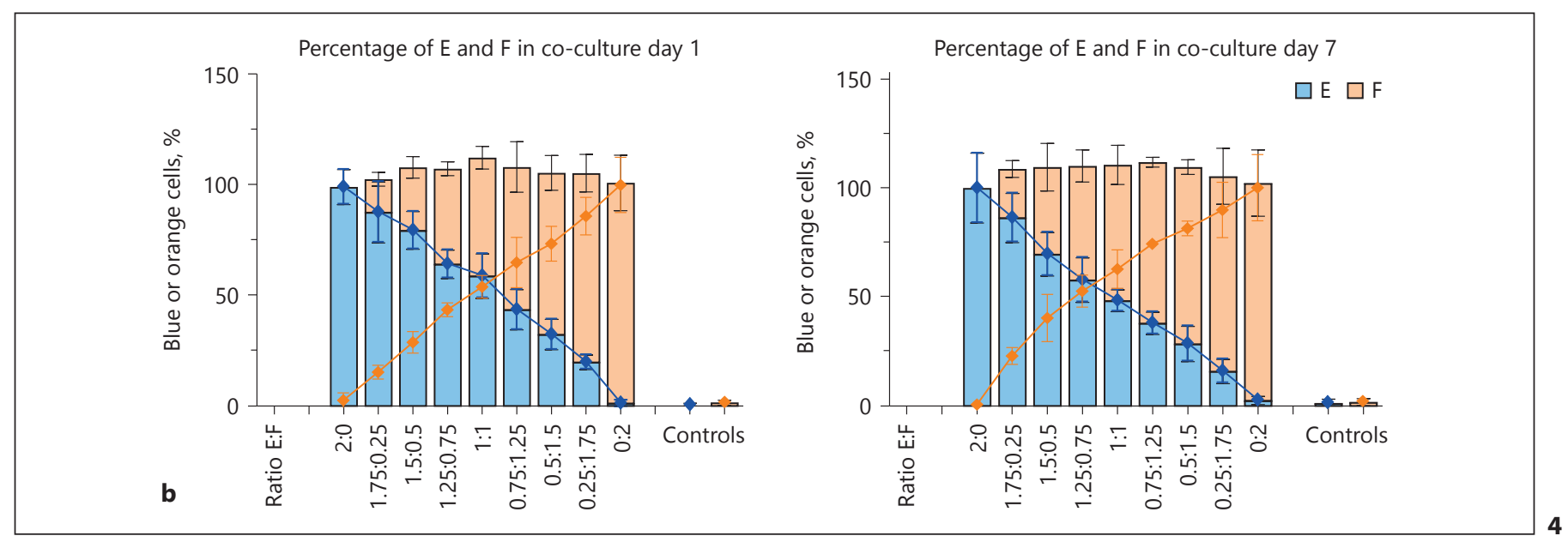

Established Fibrotic Pathways Contribute to Enhanced ECM Accumulation during Fibroblast: Epithelial Cell Contact Co-Culture

To understand further whether the phenomenon of increased ECM accumulation in contact co-culture is likely to represent a physiologically relevant process, we tested the contribution of well-established fibrotic pathways to ECM accumulation. Initially, we tested nintedanib, a small molecule tyrosine-kinase inhibitor, targeting vascular endothelial growth factor receptor (VEGFR), fibroblast growth factor receptor (FGFR) and platelet-derived growth factor receptor (PDGFR). Nintedanib is a clinically proven anti-fibrotic for the treatment of IPF that has been shown to reduce matrix production $[18,19]$. Nintedanib did induce significant cell cytotoxicity at high concentrations as read-out using the cell viability signal, but significant inhibition of total ECM accumulation was observed at concentrations that maintained cell viability (Fig. 6a). Similar inhibitory data were obtained with the other ECM markers, fibronectin and collagens, which are summarized in Table 2. It should be noted that nintedanib was also a potent inhibitor of a lung small airway epithelial cell: IPF lung fibroblast co-culture ECM accumulation assay (data not shown), at similar concentrations demonstrating activity in the current study.

TGF- $\beta$ is a master regulator of fibrosis [20], so we also sought to test whether it was contributing to ECM accumulation in our cell system. Consistent with our previous study, we observed that fresolumimab, a pan TGF- $\beta$ antibody with an anti-fibrotic effect in clinical trials [21,22], significantly inhibited ECM accumulation in a dose-dependent manner (Fig. 6b; Table 3).

Role of Integrins in a Human in vitro Model of Renal Fibrosis
Integrins Make a Major Contribution to Enhanced ECM Accumulation during Fibroblast: Epithelial Cell Contact Co-Culture

Integrins are a major family of proteins that contribute to fibrosis through a number of mechanisms including TGF- $\beta$ activation [23]. We therefore sought to determine whether integrins could play a role in the ECM accumulation driven by the interaction between fibroblasts and epithelial cells.

Initially, we investigated the presence of multiple integrins in both the mono- and co-culture cell systems by looking at their expression at the mRNA level. A microarray experiment was conducted comparing the co-culture of epithelial cells and fibroblasts with the respective monocultures, in order to determine which integrins, if any, were differentially regulated in the co-culture model system. The heat map shows the transcript levels of all 23 different integrin ( $15 \alpha$-chains and $8 \beta$-chains) subunits in both the mono-cultures of epithelial cells and fibroblasts and in the co-culture at $48 \mathrm{~h}$ (Fig. 7a). There was no significant change in expression of any of the integrin subunits in the co-culture compared to either cell type in mono-cultures ( $48 \mathrm{~h}$ time point). Also noted was the wide variation in expression levels comparing across the family; the integrin $\alpha \mathrm{V}$ subunit (coded for by the ITGAV gene) and its preferential binding partners subunits $\beta 1, \beta 5$ and $\beta 6$ (coded for by ITGB1, ITGB5 and ITGB6 genes, respectively) are highly expressed across all cultures. Other highly expressed $\alpha$-integrin subunits are $\alpha 3, \alpha 4, \alpha 5, \alpha 6$ and $\alpha \mathrm{E}$ (coded for by ITGA3, ITGA4, ITGA5, ITGA6 and ITGAE genes, respectively) with very little differences again between mono- and co-cultures. The full set of integrin gene expression profiles are summarized in Table 4. 
The expression of the $\alpha v, \beta 1, \beta 5, \beta 6$ and $\beta 8$ subunits at the protein level was then assessed using flow cytometry. Interestingly, the levels of integrin $\alpha \mathrm{V}$ were much higher in the epithelial cells than the fibroblasts, whereas the $\beta 1$ subunit was highly expressed in both cell types, and $\beta 5$, $\beta 6$ and $\beta 8$ were only present at lower levels or were undetectable (Fig. 7b). Images of the colour-coded cells in coculture confirmed the higher amounts of integrin $\alpha \mathrm{V}$ in the epithelial cells compared to the fibroblasts, the high amounts of integrin $\beta 1$ in both cell types, and the nondetectable levels of integrin $\beta 5, \beta 6$ and $\beta 8$ in all cultures (online suppl. Fig. S5).

Using a range of blocking antibodies, we tested the contribution of a number of integrins to ECM accumulation. It was found that the anti-integrin $\alpha \mathrm{V} \beta 5$ and the anti-integrin $\alpha \mathrm{V} \beta 6$ antibodies did show some inhibition of matrix accumulation, even though doses of 1,000 nM were necessary to demonstrate significant inhibition. The pan anti-integrin $\alpha \mathrm{V}$ antibody, MABT207, showed a significant inhibitory effect at both 10 and 1,000 nM (Fig. 8a) with IC50s in the sub-nanomolar range on both total ECM and individual ECM components (Fig. 8bi; Table 5). It was noted that even when the plateau of inhibition of ECM accumulation by the potent anti-alpha $\mathrm{V}$ antibody had been reached, there was still a low level of ECM remaining in the well (Fig. 8bii). This was similar to that observed in the individual mono-cultures (Fig. 1a) and represents the maximum inhibitory signal out of all the inhibitory test reagents that were trialled in this study, thus demonstrating that alpha $\mathrm{V}$ integrin-dependent mechanisms are critical in regulating the co-culture ECM response. Nevertheless, these data do not rule out other regulatory mechanisms participating with $\alpha \mathrm{V}$ integrins as mediators of ECM accumulation during fibroblast: epithelial cell interactions.

Having previously observed the significant contribution of TGF- $\beta$ and integrin $\alpha \mathrm{V}$ to ECM accumulation, it was then tested whether a combination of neutralising both TGF- $\beta$ and integrin $\alpha \mathrm{V}$ would result in more potent blockade. It was found that there was no additive or synergistic effect observed with this combination together, suggesting that both TGF- $\beta$ and $\alpha \mathrm{V}$ were on sequential rather than parallel pathways (online suppl. Fig. S6). The large differential potency between blocking the TGF- $\beta$ and $\alpha \mathrm{V}$ pathways (IC50 139.5 and $0.533 \mathrm{nM}$, respectively), highlights the central role of $\alpha \mathrm{V}$ integrins in mediating the signalling events leading to ECM accumulation and could imply that $\alpha \mathrm{V}$ interactions with binding partners could be involved in other mechanisms apart from activation of latent TGF- $\beta$. It should be noted that the respec- tive described effective IC50 inhibitory concentrations for the MAB1835 $(0.25-1.25 \mu \mathrm{g} / \mathrm{mL})$ and MABT207 (0.1 $\mu \mathrm{g} / \mathrm{mL})$ in cell assays are comparable $[24,25]$ and in a lung epithelial: fibroblast co-culture, total ECM was potently inhibited by both the anti-TGF- $\beta$ antibody (IC50 $10.01 \mathrm{nM}$; data not shown) and the anti-integrin $\alpha \mathrm{V}$ antibody (IC50 $0.57 \mathrm{nM}$; data not shown) which we concluded was due to the dominant signalling pathways varying between the different organ co-cultures.

\section{Integrin Small Molecule Inhibitors are as Effective as}

Nintedanib in Blocking ECM Accumulation

Nanthakumar et al. [16] inventoried all the anti-fibrotic small molecules currently being tested for kidney, lung, liver and skin diseases. From this list, a selected set of 26 small molecule inhibitors, based on their commercial availability and their predicted anti-fibrotic effect in kidney disease, were examined in our renal co-culture cell system. The effect of these inhibitors on the total ECM proteins in the renal co-culture system was tested at the highest dose before toxicity was observed. Compounds were organised based on the category of molecular target (i.e., cytokines, receptors, ion channels, kinases, signalling molecules, enzymes and integrins). Out of the 26 exploratory compounds tested, only 5 showed significant inhibition of matrix accumulation. They were SB523334, an ALK 5 inhibitor (the receptor complex that mediates the signalling of TGF- $\beta$ ); CWHM12, a pan integrin $\alpha \mathrm{V}$ inhibitor; $\mathrm{C} 8$, an integrin $\alpha \mathrm{V} \beta 1$ inhibitor; SB273005, an integrin $\alpha V \beta 3 / \alpha V \beta 5$ inhibitor and DC9711, an integrin $\alpha \mathrm{V} \beta 6$ inhibitor (Fig. 9). Four out of the set of 26 molecules were integrin inhibitors demonstrating significant inhibition of total ECM, and the most potent compound, CWHM12, a pan $\alpha \mathrm{V}$ integrin inhibitor showed equivalent knockdown of the total ECM to the clinical candidate, nintedanib. The more specific integrin blockers targeting the heterodimers, $\alpha \mathrm{V} \beta 1, \alpha \mathrm{V} \beta 3 / \beta 5$ and $\alpha \mathrm{V} \beta 6$ all showed significant inhibition, but there was remaining total ECM that could not be inhibited and the effect of higher concentrations could not be determined due to cell toxicity effects. It was noted that cilengitide, the $\alpha \mathrm{V} \beta 3$ and $\alpha \mathrm{V} \beta 5$ inhibitor, which demonstrated inhibition in a kidney mouse systemic sclerosis model [11] was shown to have no significant effect in the current renal assay in vitro model. The differential responses of the $2 \alpha \mathrm{V} \beta 3 / 5$ inhibitors may be due in part to differential potencies for the target where the $\mathrm{K}_{\mathrm{i}}$ of SB273005 is 0.3 and $1.2 \mathrm{nM}$ for $\alpha \mathrm{V} \beta 5$ and $\alpha \mathrm{V} \beta 3$, respectively, and cilengitide the $K_{i}$ is 4.1 and $79 \mathrm{nM}$ for $\alpha \mathrm{V} \beta 3$ and $\alpha \mathrm{V} \beta 5$, respectively $[26,27]$, and in part due to cellular toxicity limiting 


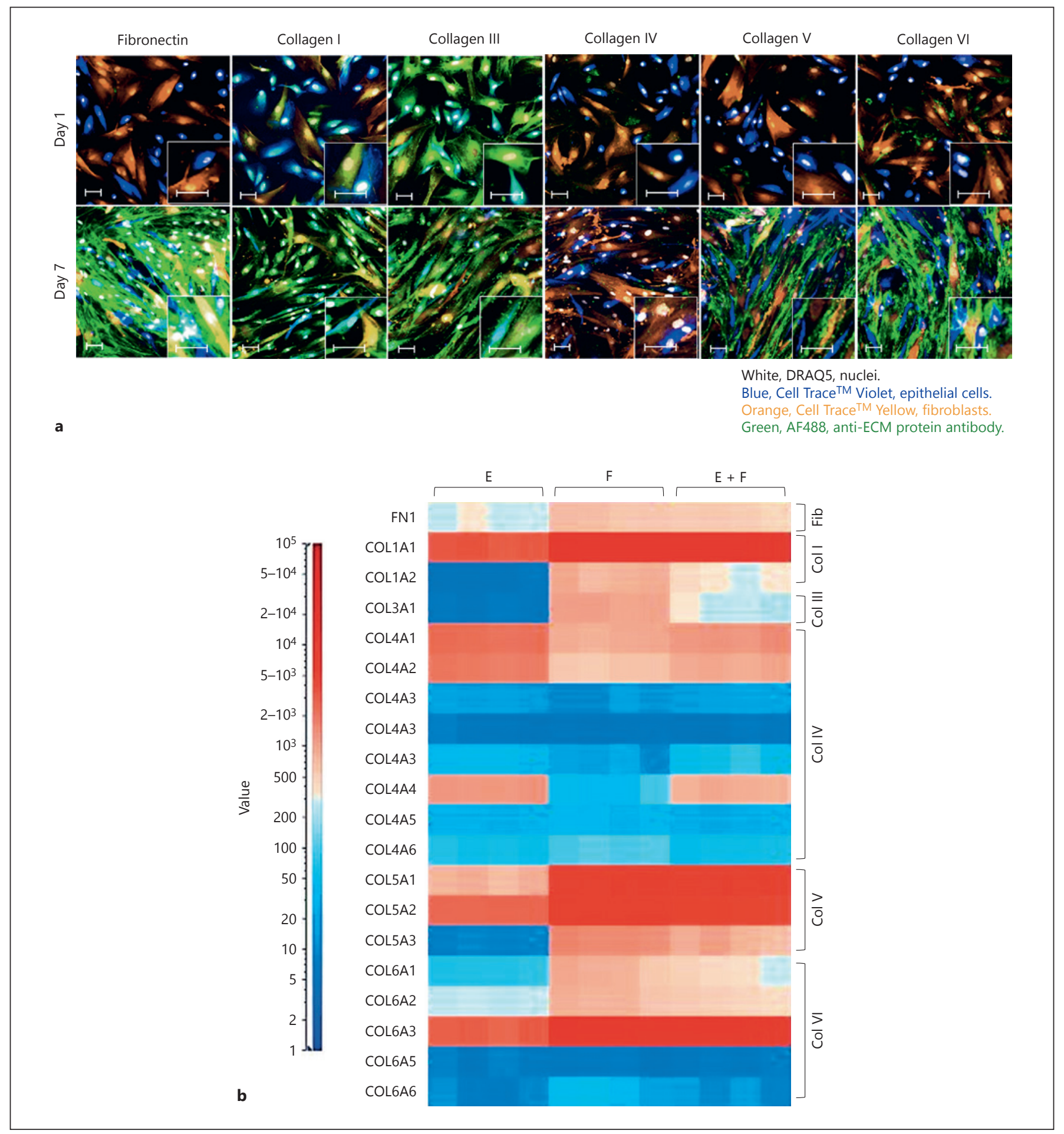

Fig. 5. Both epithelial cells and fibroblasts contribute to the accumulation of ECM. a Images show immunofluorescent staining of the epithelial cells (Cell Trace ${ }^{\mathrm{TM}}$ Violet, blue) and fibroblasts (Cell Trace $^{\mathrm{TM}}$ Yellow, orange) for fibronectin, collagens I, III, IV, V or VI (green) after 1 or 7 days in co-culture. b Epithelial cells and fibroblasts were cultured for $48 \mathrm{~h}$ in mono-culture or co-culture. RNAs were then extracted and transcript expression levels were assessed using Affymetrix Bead Arrays. The heat map shows the expression levels of a panel of ECM genes in the mono-cultures and the co-culture. Results are summarised in Table 1, signal value $(-)<30 ;(+)$ 30-100; (++) 100-1,000; (+++) >1,000. E, mono-culture of epithelial cells; F, mono-culture of fibroblasts; E + F, coculture of epithelial cells and fibroblasts. Images show a single field. Scale bars represent $50 \mu \mathrm{m}$. ECM, extracellular matrix. 
Table 1. Expression levels of a panel of ECM genes in the mono-cultures and co-culture of epithelial cells and fibroblasts

\begin{tabular}{|c|c|c|c|c|c|}
\hline Collagen & Gene symbol & Gene title & $\mathrm{E}$ & $\mathrm{F}$ & $E+F$ \\
\hline & FN1 & Fibronectin, 1 & ++ & ++ & ++ \\
\hline \multirow[t]{2}{*}{ I } & COL1A1 & Collagen, type I, alpha 1 & +++ & +++ & +++ \\
\hline & COL1A2 & Collagen, type I, alpha 2 & - & ++ & ++ \\
\hline III & COL3A1 & Collagen, type III, alpha 1 & - & +++ & ++ \\
\hline \multirow[t]{6}{*}{ IV } & COL4A1 & Collagen, type IV, alpha 1 & +++ & ++ & +++ \\
\hline & COL4A2 & Collagen, type IV, alpha 2 & +++ & ++ & ++ \\
\hline & COL4A3 & Collagen, type IV, alpha 3 & - & - & - \\
\hline & COL4A4 & Collagen, type IV, alpha 4 & +++ & + & ++ \\
\hline & COL4A5 & Collagen, type IV, alpha 5 & - & - & - \\
\hline & COL4A6 & Collagen, type IV, alpha 6 & + & + & + \\
\hline \multirow[t]{3}{*}{$\mathrm{V}$} & COL5A1 & Collagen, type $V$, alpha 1 & ++ & +++ & +++ \\
\hline & COL5A2 & Collagen, type $\mathrm{V}$, alpha 2 & +++ & +++ & +++ \\
\hline & COL5A3 & Collagen, type $V$, alpha 3 & - & +++ & ++ \\
\hline \multirow[t]{5}{*}{ VI } & COL6A1 & Collagen, type VI, alpha 1 & + & ++ & ++ \\
\hline & COL6A2 & Collagen, type VI, alpha 2 & ++ & ++ & ++ \\
\hline & COL6A3 & Collagen, type VI, alpha 3 & +++ & +++ & +++ \\
\hline & COL6A5 & Collagen, type VI, alpha 5 & - & - & - \\
\hline & COL6A6 & Collagen, type VI, alpha 6 & - & - & - \\
\hline
\end{tabular}

ECM, extracellular matrix.

the maximum concentration of cilengitide employed in the assay. The known binding partners for $\alpha \mathrm{V}$ integrin are $\beta 1, \beta 3, \beta 5, \beta 6$ or $\beta 8$, but unfortunately, the contribution of the $\beta 8$ subunit to the ECM response could not be explored due to the lack of a specific inhibitor.

Comparing compounds side by side in the same assay, only the pan $\alpha \mathrm{V}$ integrin inhibitor, CWHM12 at $1 \mu \mathrm{M}$ and the ALK5 inhibitor, SB525334 at $10 \mu \mathrm{M}$ were able to replicate the maximum effect of nintedanib at $1 \mu \mathrm{M}$, using non-toxic doses, in reducing the ECM accumulation in the renal co-culture system. The potent effect of both the small molecule $\alpha \mathrm{V}$ integrin inhibitor and the anti-aV monoclonal antibody (MABT207) highlights the $\alpha \mathrm{V}$ integrin family of cellular adhesion molecules as key regulators of ECM accumulation in a functionally relevant in vitro fibrosis assay. This could help to highlight these molecules as starting points for future clinical candidates.

The other marketed anti-fibrotic candidate, pirfenidone was toxic at doses higher than $10 \mu \mathrm{M}$ and no inhibition of the ECM response could be detected at non-toxic doses (Fig. 9).
The non-active inhibitors in the renal co-culture assay target a number of different target classes that have been reported in the literature as potential anti-fibrotic renal mechanisms. Examples of these mechanisms included in this screen are (1) CCL2/CCR2 inhibitors (bindarit and RS504393); (2) receptor agonists such as prostacyclin (beraprost sodium), PPAR (IVA337), 5H2A (sarpogrelate hydrochloride), FXR (obeticholic acid), S1P (FTY720) and lipoxin (BML-111); (3) potassium channel blocker (TRAM34); (4) multi-kinase inhibitors of Bcrabl, kit and PDGFR (nilotinib) and Jak1/2 (baricinib); (5) modulators of signalling molecules such as mTOR (rapamycin) and oxidative glycation (pyridoxamine dihydrochloride) and (6) enzyme inhibitors such as autotaxin (L3223), angiotensin-converting enzyme (enalapril maleate) and caspases (emricasam). The lack of inhibition with these molecules does not preclude their importance in fibrotic mechanisms but potentially excludes a role for these pathways in the current renal co-culture ECM accumulation system. 


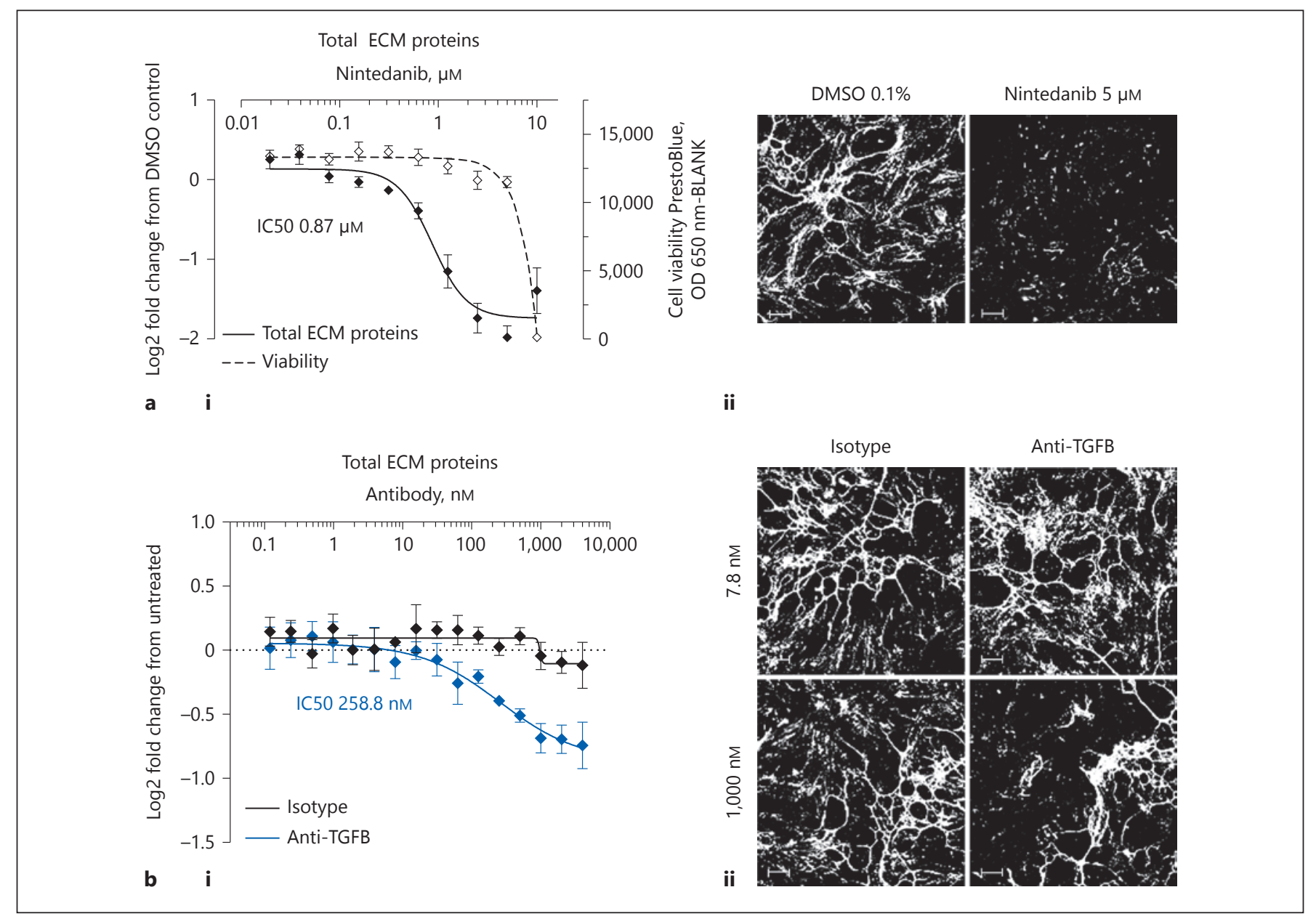

Fig. 6. Clinically proven anti-fibrotics, Nintedanib and Fresolumimab, effectively inhibit ECM accumulation. a Effect of a marketed anti-fibrotic, Nintedanib, on ECM in the co-culture of epithelial cells and fibroblasts. Cells were cultured for 7 days in coculture in the presence of Nintedanib (10-0.02 $\mu \mathrm{M})$. Cells were then lysed, and the ECM was fixed and stained using a total protein dye or specific antibodies. i Graph shows the effect of Nintedanib on total ECM components (total protein dye, solid line) or on cell viability (PrestoBlue, dashed line). Data are represented as $\log 2$ fold change from the co-culture in the presence of $0.1 \%$ DMSO and shows mean \pm SD from a representative of 3 independent experiments with 4 replicate wells per condition. IC50s estimated for the total and individual ECM markers from the independent experiments are presented as geomeans with the range in Table 2. ii Corresponding images of total ECM components (total protein dye, white) for $5 \mu \mathrm{M}$ Nintedanib or DMSO control $(0.1 \%$ DMSO). $\mathbf{b}$ Effect of an anti-TGFB antibody on ECM accumula-

\section{Discussion}

The interaction of multiple cell types is likely to play an important role in the deposition of ECM in fibrosis $[5,7,28,29]$. We observed a phenomenon whereby tion in the co-culture of epithelial cells and fibroblasts. Cells were cultured for 7 days in the presence of anti-TGFB antibody (MAB1835) or isotype control (MAB002; 4,000-0.12 nM). Cells were then lysed, and the ECM was fixed and stained using a total protein dye or specific antibodies. i Graph shows the effect of the anti-TGFB antibody (in blue) on total ECM. Corresponding isotype control is also shown (in black). There was no effect on cell viability (PrestoBlue) at any concentration tested. Data are represented as $\log 2$ fold change from the untreated co-culture and show mean \pm SD from a representative of 4 independent experiments with 4 replicate wells per condition. IC50s estimated for the total and individual ECM markers from the independent experiments are summarised as geomeans with the range in Table 3. ii Corresponding images of total ECM components (total protein dye, white) for 7.8 or 1,000 nM anti-TGFB antibody or isotope control. Images show a single field. Scale bars represent $100 \mu \mathrm{m}$. ECM, extracellular matrix. mary renal fibroblasts in 2D culture triggers large increases in mature ECM accumulation. Under resting conditions in mono-culture, as expected, epithelial cells and fibroblasts expressed low intracellular levels of fi- 
Table 2. IC50s for nintedanib in ECM accumulation assay for total and individual ECM markers

\begin{tabular}{llll}
\hline & $\begin{array}{l}\text { IC50 geomean, } \\
\mu \mathrm{M}\end{array}$ & $\begin{array}{l}\text { Nintedanib, } \\
\text { range, } \mu \mathrm{M}\end{array}$ & Number \\
\hline Total ECM proteins & 0.672 & $0.579-0.865$ & 3 \\
Fibronectin & 0.882 & $0.797-1.273$ & 3 \\
Collagens I and III & 0.421 & $0.134-0.855$ & 3 \\
Collagen IV & 0.704 & $0.244-1.252$ & 3 \\
Collagen V & 0.326 & $0.193-0.440$ & 3 \\
\hline
\end{tabular}

ECM, extracellular matrix

Table 3. IC50s for the pan TGF- $\beta$ antibody, Fresolumimab in ECM accumulation assay for total and individual ECM markers

\begin{tabular}{llcl}
\hline & $\begin{array}{c}\text { Geomean } \\
\text { IC50, nM }\end{array}$ & Range, nM & Number \\
\hline Total ECM proteins & 236.6 & $126.8-490$ & 4 \\
Fibronectin & 158.0 & $124-202$ & 4 \\
Collagens I and III & 187.6 & $131.3-215.1$ & 4 \\
Collagen IV & 255.5 & $119.3-1,614$ & 4 \\
Collagen V & 118.2 & $86.1-160.6$ & 4 \\
\hline
\end{tabular}

ECM, extracellular matrix; TGF- $\beta$, transforming growth factor- $\beta$.

bronectin and the fibrillar collagens IV, V and VI at the protein level, with the exception of collagens I and III, which appeared to be constitutively present in monocultures. However, following contact co-culture, both cell types showed increased staining for intracellular collagens. An exception was collagen IV where low expression was also observed even in the co-culture. In addition to cell accumulation of matrix proteins, a pronounced ECM accumulation was also observed when cells were stripped and the matrix imaged, highlighting a level of regulation occurring in the export and extracellular accumulation of these matrix proteins. This is particularly exemplified with collagens I and III where the protein is clearly present in the individual cell types in mono-culture but detected at low levels in the extracellular space; in the co-culture format, collagens I and III significantly accumulate in the ECM, validating the assay format for ECM measurements. Interestingly, the spontaneous ECM accumulation appears to be a generic phenomenon, which we have observed with primary epithelial cells and fibroblast co-cultures across a number of different organ systems including lung, liver and skin (data not shown). The exact molecular mechanisms driving the different organ ECM responses are currently under investigation.

The 2D primary human renal epithelial and fibroblast co-culture in vitro model described could be used in the hunt for new anti-fibrotic renal therapeutics or in the characterization of the mode of action of known candidates. There are 2 alternative human renal epithelial and fibroblast co-culture models that have been characterized for similar purposes. One such 3D model uses a human immortalized dermal fibroblast embedded in a collagen gel with a RPTEC line layered on top and then challenged with the toxin cisplatin to induce epithelial cell injury [5]. Gene expression was then performed to assess transition to the activated myofibroblast phenotype. The second model is also a 3D non-contact co-culture, but the proximal tubular epithelial cell line is allowed to form spheroids within a dextran hydrogel and then again overlaid with a renal fibroblast cell line in a similar hydrogel [30]. Nephrotoxic compounds are applied to cause epithelial injury, and the read-outs comprise of image-based characterization of the epithelial cells and myofibroblast differentiation. Although there are many similarities in these in vitro cell models with our co-culture model in that both use a combination of epithelial cells and fibroblasts, there are also a number of key differences that make the assay described here potentially of more translational relevance. In our model, the 2 cell types are in direct contact co-culture, whereas both the Moll et al. [5] and Nugraha et al. [30] models have cells in separate communicable layers. Further the model described here uses primary human cells as opposed to immortalized cells while the activation of a fibrotic phenotype is induced spontaneously via cell contact instead of toxin-induced epithelial damage. However, the main and most important difference is the read-out on mature ECM accumulation, which is independent of contamination by unprocessed cellular ECM proteins. We believe this provides a direct link to fibrotic pathobiology as it reads out on the final product of the scarring process directly rather than a surrogate and thus encompasses changes in all homeostatic processes that regulate this. In both the Moll et al. [5] and Nugraha et al. [30] co-cultures systems, the read-outs such as epithelial cell injury, myofibroblast activation and modulation of kidney molecular toxicity gene expression panels, while valuable, only represent specific parts of the larger fibrotic pathobiology and are all upstream of mature ECM levels. The importance of this is reinforced by the data presented here, which indicates that changes in "fi- 
Fig. 7. Multiple integrins are expressed in the mono-cultures and co-culture of epithelial cells and fibroblasts. a Epithelial cells and fibroblasts were cultured for $48 \mathrm{~h}$ in mono-culture or co-culture. RNAs were then extracted, and transcript expression levels were assessed using Affymetrix Bead Arrays. The heat map shows the expression levels of the integrin $\alpha$-chains and $\beta$-chains in the mono-cultures and the co-culture. Results are summarised in Table 4, signal value $(-)<30$; (+) 30-100; (++) 100-1,000; $(+++)>1,000$. b Flow cytometric analysis of integrin alpha $\mathrm{V}$, integrin beta $1,5,6$ and 8 in primary human tubular epithelial cells and fibroblasts. Left panel shows overlay of FACS histogram obtained for the different integrins in the epithelial cells. Right panel shows the same for the fibroblasts. Corresponding isotype control is shown in dark grey.

Role of Integrins in a Human in vitro Model of Renal Fibrosis

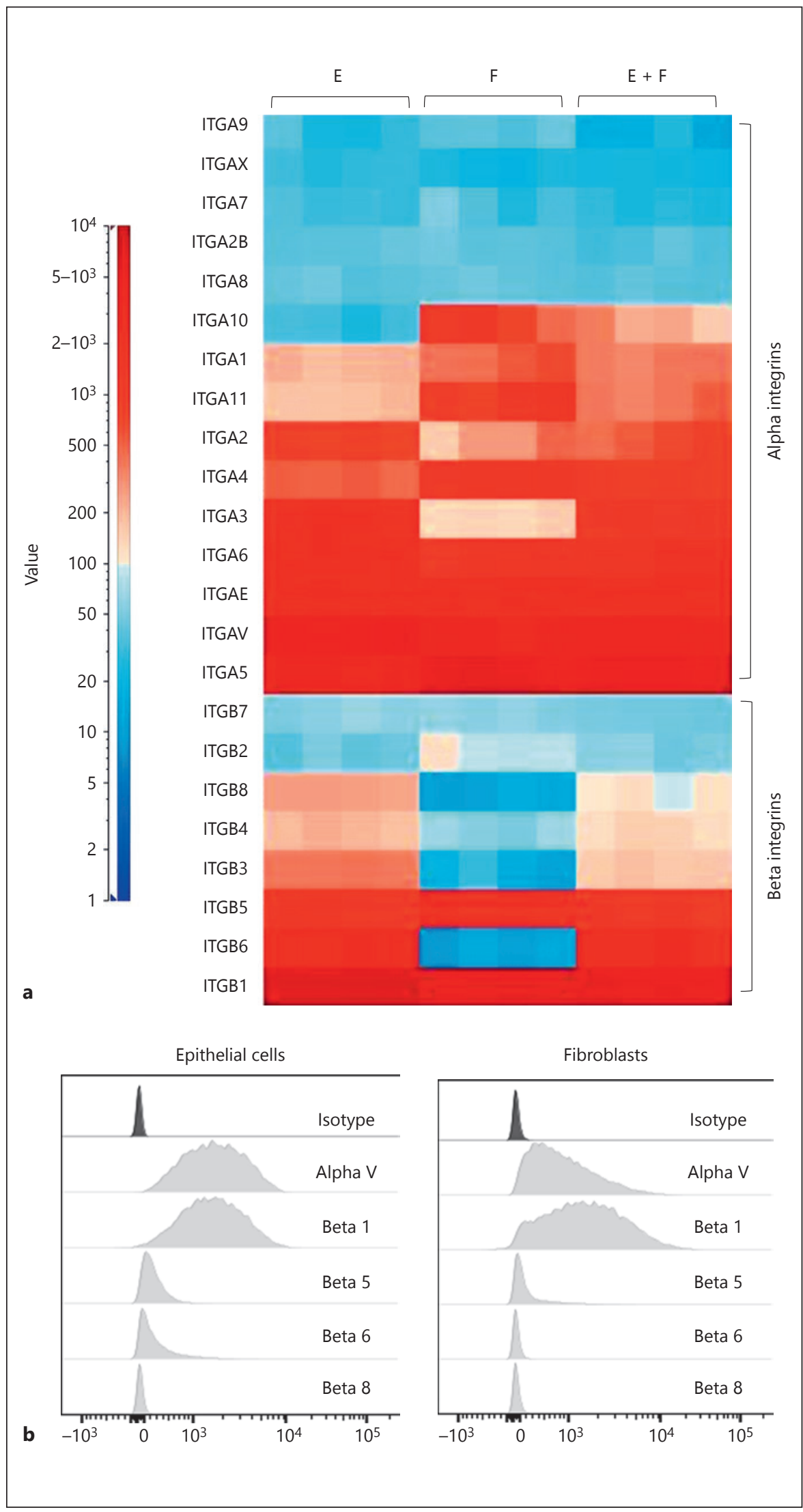


Table 4. Expression levels of $\alpha$ and $\beta$-integrin subunits genes in the mono-cultures and co-culture of epithelial cells and fibroblasts

\begin{tabular}{|c|c|c|c|c|}
\hline Gene symbol & Gene title & $\mathrm{E}$ & $\mathrm{F}$ & $E+F$ \\
\hline \multicolumn{5}{|l|}{ Alpha subunits } \\
\hline ITGAV & Integrin alpha $\mathrm{V}$ & +++ & +++ & +++ \\
\hline ITGA1 & Integrin alpha 1 & ++ & ++ & ++ \\
\hline ITGA2 & Integrin alpha 2 & ++ & ++ & ++ \\
\hline ITGA3 & Integrin alpha 3 & +++ & ++ & +++ \\
\hline ITGA4 & Integrin alpha 4 & ++ & +++ & +++ \\
\hline ITGA5 & Integrin alpha 5 & +++ & +++ & +++ \\
\hline ITGA6 & integrin alpha 6 & +++ & +++ & +++ \\
\hline ITGA7 & Integrin alpha 7 & + & + & + \\
\hline ITGA8 & Integrin alpha 8 & + & + & + \\
\hline ITGA9 & Integrin alpha 9 & + & + & + \\
\hline ITGA10 & Integrin alpha 10 & + & ++ & ++ \\
\hline ITGA11 & Integrin alpha 11 & ++ & +++ & ++ \\
\hline ITGAD & Integrin alpha D & - & - & - \\
\hline ITGAE & Integrin alpha $\mathrm{E}$ & +++ & +++ & +++ \\
\hline ITGAL & Integrin alpha L & - & - & - \\
\hline ITGAM & Integrin alpha $\mathrm{M}$ & - & - & - \\
\hline ITGAX & Integrin alpha $\mathrm{X}$ & + & + & + \\
\hline ITGA2B & Integrin alpha $2 \mathrm{~b}$ & + & + & + \\
\hline \multicolumn{5}{|l|}{ Beta subunits } \\
\hline ITGB1 & Integrin beta 1 & +++ & +++ & +++ \\
\hline ITGB2 & Integrin beta 2 & + & + & + \\
\hline ITGB3 & Integrin beta 3 & ++ & + & ++ \\
\hline ITGB4 & Integrin beta 4 & ++ & + & + \\
\hline ITGB5 & Integrin beta 5 & +++ & +++ & +++ \\
\hline ITGB6 & Integrin beta 6 & +++ & + & +++ \\
\hline ITGB7 & Integrin beta 7 & + & + & + \\
\hline ITGB8 & Integrin beta 8 & ++ & + & ++ \\
\hline
\end{tabular}

brotic ECM genes" at the transcriptional level do not necessarily reflect changes in the ECM accumulation of the corresponding protein. Finally, our work has also shown that epithelial cells alongside activated fibroblasts are capable of directly contributing to ECM accumulation to a significant level. This was also demonstrated in a previous publication where TGF- $\beta$-stimulated primary human renal tubular epithelial cells induced fibrillar collagens and fibronectin to very similar levels as seen in similar treated primary HRFs [7].

At the outset of this study, we hypothesised that, along with the central pro-fibrotic mediator TGF- $\beta$, the $\alpha \mathrm{V}$ integrin family could play a key role in mediating the renal contact co-culture ECM accumulation signal. TGF- $\beta$ is synthesized in a precursor form, which is proteolytically cleaved to release latency associated peptide (LAP) that binds to the mature TGF- $\beta$ homodimer. The LAP of TGF- $\beta 1$ and TGF- $\beta 3$ contains a linear arginineglycine-aspartic acid (RGD) motif, which can interact with any of the $\alpha \mathrm{V}$ integrin family $(\alpha \mathrm{V} \beta 1, \alpha \mathrm{V} \beta 3, \alpha \mathrm{V} \beta 5$, $\alpha \mathrm{V} \beta 6$ and $\alpha \mathrm{V} \beta 8$ ), resulting in its activation. Indeed, we have shown using a blocking pan anti- $\alpha \mathrm{V}$ integrin antibody, anti- $\alpha \mathrm{V} \beta 5$ and anti- $\alpha \mathrm{V} \beta 6$ specific blocking antibodies, as well as a number of small molecule integrin inhibitors, that the spontaneous ECM signal is substantially reduced in the presence of some of these inhibitors. The more potent blockers in terms of maximum inhibitory signals were the pan $-\alpha \mathrm{V}$ integrin inhibitors that blocked the full range of heterodimers $(\alpha \mathrm{V} \beta 1, \alpha \mathrm{V} \beta 3$, $\alpha \mathrm{V} \beta 5, \alpha \mathrm{V} \beta 6$ and $\alpha \mathrm{V} \beta 8$ ) such as the MABT207 monoclonalantibody and the small moleculeinhibitor, CWHM12. Significant inhibition was also observed with the antiTGF- $\beta$ blocking antibody (even though very high levels of antibody were required) and the small molecule ALK5 inhibitor, SB525334. Testing if there was any advantage in combining the anti-pan $\alpha \mathrm{V}$ and anti-TGF- $\beta$ blocking antibodies demonstrated no additional efficacy, implying that these targets were more likely to be arranged in 


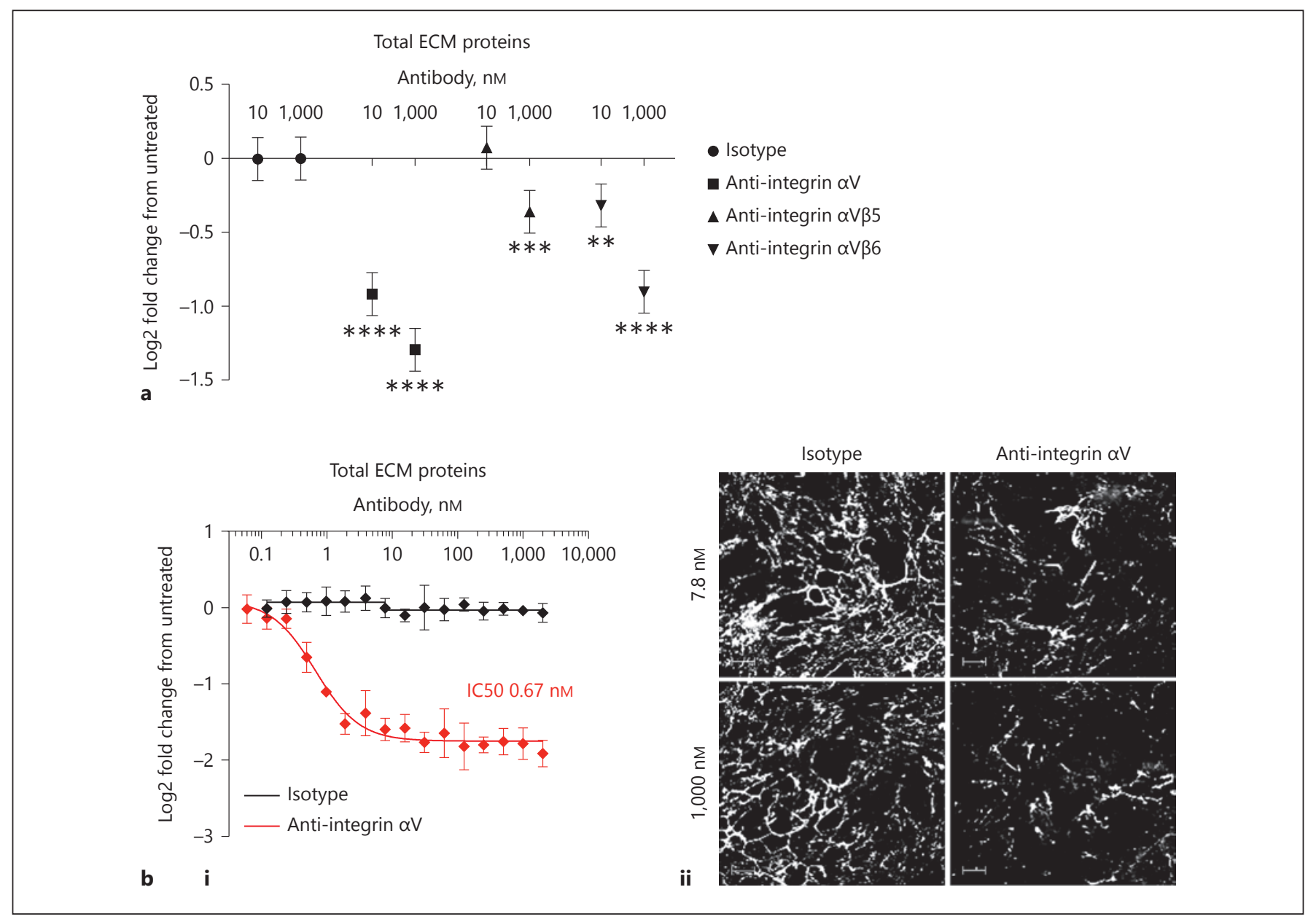

Fig. 8. Integrins make a major contribution to enhanced ECM accumulation during fibroblast: epithelial cell contact co-culture. a Effect of anti-integrin antibodies on ECM accumulation in the coculture of epithelial cells and fibroblasts. Cells were cultured for 7 days in the presence of anti-integrin $\alpha \mathrm{V}$ (MABT207), anti-integrin $\alpha$ V $\beta 5$ (MAB2528) and anti-integrin $\alpha$ V $\beta 6$ (ab77906) antibodies or isotype control (MAB002; 10 or 1,000 nM). ECM was stained as described above. Graph shows the effect of the antibodies on total ECM. Data are represented as $\log 2$ fold change from the untreated co-culture and show mean with 95\% CIs from 3 independent experiments with 4 replicate wells per condition. b Effect of an anti-integrin $\alpha \mathrm{V}$ antibody on ECM accumulation in the coculture of epithelial cells and fibroblasts. Cells were cultured for 7 days in the presence of anti-integrin $\alpha \mathrm{V}$ antibody (MABT207) or isotype control (MAB002; 2,000-0.06 nM). ECM was stained as described above. $\mathbf{i}$ Graph shows the effect of the anti-integrin $a \mathrm{~V}$

sequence. Drilling down to the specific integrin heterodimer that is responsible for mediating the contact co-culture ECM signal was hampered due to the lack of a comprehensive set of reagents, but the selective $\alpha \mathrm{V} \beta 1$ inhibitor (C8), $\alpha \mathrm{V} \beta 3 / \beta 5$ inhibitor (SB273005) and $\alpha \mathrm{V} \beta 6$ antibody (in red) on total ECM. Corresponding isotype control is also shown (in black). There was no effect on cell viability (PrestoBlue) at any concentration tested. Data are represented as log2 fold change from the untreated co-culture and show mean \pm SD from a representative of 4 independent experiments with 4 replicate wells per condition. The estimated IC50s for the independent experiments are summarised as geomeans with the range in Table 5. ii Corresponding images of total ECM components (total protein dye, white) for 7.8 or 1,000 nM anti-integrin aV antibody or isotope control. E, mono-culture of epithelial cells; F, mono-culture of fibroblasts; E + F, co-culture of epithelial cells and fibroblasts. Images show a single field. Scale bars represent $100 \mu \mathrm{m} .{ }^{*} p<0.05$, ${ }^{* *} p<0.01,{ }^{* * *} p<0.001,{ }^{* * * *} p<0.0001$; Analysis of variance fitting dose and group, allowing for the different experiments and comparison of each group to dose-matched isotype control (a). ECM, extracellular matrix.

(DC9711) at their maximum tolerated concentrations, all showed significant inhibition of the co-culture ECM, but were unable to show maximum signal inhibition compared to the assay control, nintedanib or the pan $\alpha \mathrm{V}$ inhibitor CWHM12. Also, the contribution of $\alpha \mathrm{V} \beta 8$ 
Table 5. IC50s for the pan TGF- $\beta$ antibody, the integrin $\alpha \mathrm{V}$ antibody and the combination of the pan TGF- $\beta$ and integrin $\alpha \mathrm{V}$ antibodies

\begin{tabular}{lccc}
\hline & Geomean IC50, nM & Range, nM & Number \\
\hline Anti-TGF $\beta$ & & & \\
$\quad$ Total ECM proteins & 140.5 & $109.7-181.4$ & 3 \\
Fibronectin & 165.6 & $122.6-249.2$ & 3 \\
Collagens I and III & 102.4 & $39-200$ & 3 \\
Collagen IV & 81.3 & $30.9-177.9$ & 3 \\
Collagen V & 111.6 & $84.3-195.8$ & 4 \\
Anti-integrin aV & & & 4 \\
Total ECM proteins & 0.550 & $0.418-0.67$ & 4 \\
Fibronectin & 0.905 & $0.629-1.185$ & 4 \\
Collagens I and III & 0.725 & $0.513-1.032$ & 4 \\
Collagen IV & 0.630 & $0.375-1.465$ & 3 \\
Collagen V & 0.368 & $0.317-0.451$ & 3 \\
Anti-TGF + anti-integrin $\alpha \mathrm{V}$ & & & 3 \\
Total ECM proteins & 1.096 & $0.769-1.678$ & 3 \\
Fibronectin & 1.435 & $0.965-2.157$ & 3 \\
Collagens I and III & 1.076 & $0.693-1.472$ & \\
Collagen IV & 1.348 & $1.008-1.748$ & \\
Collagen V & 0.497 & $0.439-0.596$ & \\
\hline
\end{tabular}

ECM, extracellular matrix; TGF- $\beta$, transforming growth factor $\beta$.

could not be tested as there is no specific blocker for this heterodimer. It could be concluded that all the individual $\alpha \mathrm{V}$ integrins have a significant role in their own right but collectively targeting all $\alpha \mathrm{Vs}$ together, using either the pan- $\alpha \mathrm{V}$ antibody or small molecule inhibitor, gave a more significant blockade of ECM, more akin to the level of response observed with nintedanib. Blocking of TGF- $\beta$ also had a significant effect on the overall ECM response but inhibition using the small molecule inhibitor, pirfenidone, which has also been documented as inhibitory to TGF- $\beta$ [31], had no effect; this is in contrast to the inhibitory effect demonstrated for pirfenidone on epithelial injury-induced myofibroblast activation markers [30]. We found pirfenidone to be extremely toxic in vitro at the concentrations needed to produce anti-fibrotic effects, which may explain the discrepancy between the studies or it just may be due to the different experimental models.

Precisely how this cellular interaction leads to enhanced deposition of matrix with $\alpha \mathrm{V}$ integrins and TGF- $\beta$ as central players is a key question. Cell-mediated activation of latent TGF- $\beta 1$ is a key initiating event in the promotion of fibrosis in a number of different organs [3, 32, 33]. Epithelial-associated integrin $\alpha \mathrm{V} \beta 6$ has been shown to be involved in the mediation of bleomycin-induced lung fibrosis $[34,35]$ but this was not the case for liver fi- brosis; even global deletion of all the $\alpha \mathrm{V}$ pairing $\beta$ subunits (such as $\beta 3, \beta 5$ and $\beta 8$ ) did not protect in liver fibrosis [10]. The $\alpha \mathrm{V} \beta 1$ integrin is highly expressed on activated fibroblasts and mice generated with integrin- $\beta 1$-deficient fibroblasts exhibited delayed wound closure, less granulation tissue formation (including ECM and reduced myofibroblast differentiation as shown by a-SMA expression), but these mice were not challenged in a fibrosis model setting [36]. A small molecule inhibitor, C8 that is potent and highly selective for $\alpha \mathrm{V} \beta 1\left(\mathrm{IC}_{50} 0.089 \mathrm{nM}\right)$ attenuated bleomycin-induced pulmonary fibrosis and carbon tetrachloride-induced liver fibrosis [37], suggesting that $\alpha \mathrm{V} \beta 1$ plays a critical in vivo role in tissue fibrosis. Targeting the pan $a \mathrm{~V}$ integrin genetically, using the Pdgfrb-Cre promoter in a myofibroblast-specific manner, protected mice from carbon tetrachloride-induced hepatic fibrosis, bleomycin-induced lung fibrosis and unilateral ureteral obstruction-induced kidney fibrosis [10]. In the same study, pharmacological blockade of $\alpha \mathrm{V}$ integrins using the small molecule inhibitor, CWHM12 attenuated both hepatic and lung fibrosis. In summary, there is a large weight of evidence generated from in vivo mouse models using genetically targeted approaches and therapeutics that the $\alpha \mathrm{V}$ integrin and its binding $\beta$ integrin partners have a role in mediating fibrosis that is likely to involve the activation of TGF- $\beta$. 


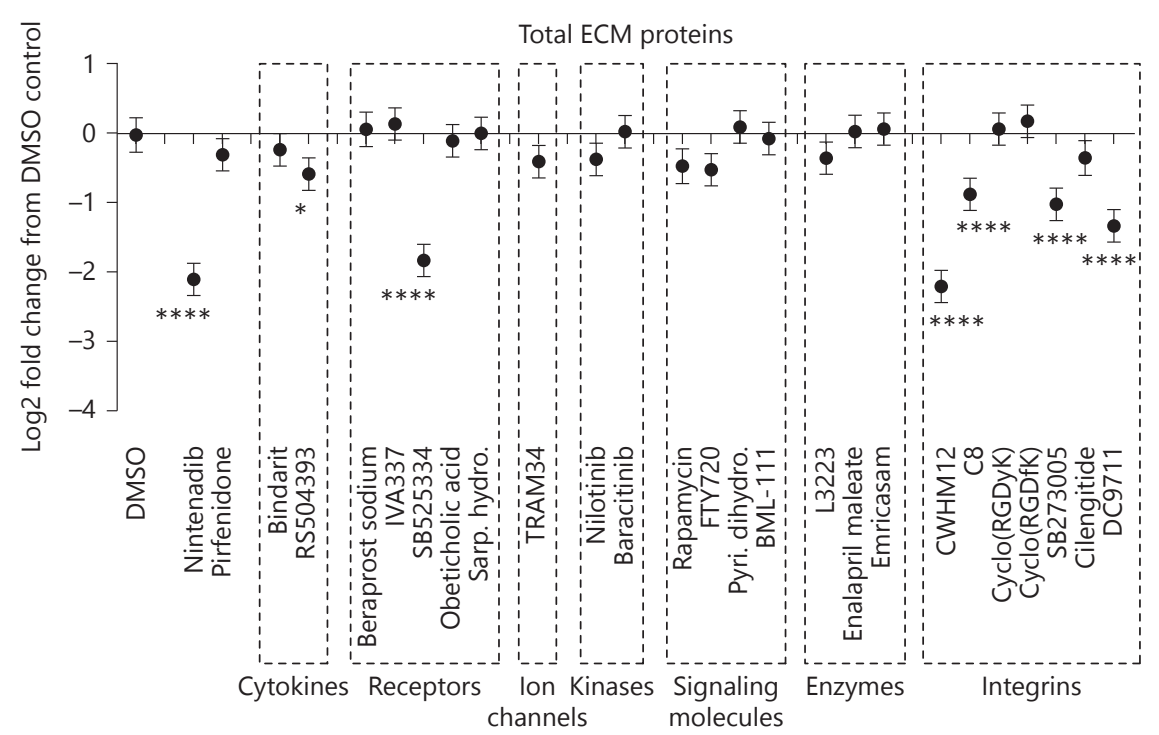

Fig. 9. Integrin small molecule inhibitors are as effective as Nintedanib in blocking ECM accumulation. Cells were cultured for 7 days in the presence of Nintedanib $(1 \mu \mathrm{M})$, Pirfenidone $(10 \mu \mathrm{M})$, Bindarit $(10 \mu \mathrm{M})$, RS504393 $(10 \mu \mathrm{M})$, Beraprost sodium $(0.3 \mu \mathrm{M})$, IVA337 $(3 \mu \mathrm{M})$, SB525334 (10 $\mu \mathrm{M})$, Obeticholic acid $(10 \mu \mathrm{M})$, Sarpogrelate hydrochloride $(1 \mu \mathrm{M})$, TRAM34 $(10 \mu \mathrm{M})$, Nilotinib $(0.3$ $\mu \mathrm{M})$, Baricitinib $(3 \mu \mathrm{M})$, Rapamycin $(10 \mu \mathrm{M})$, FTY720 $(3 \mu \mathrm{M})$, Pyridoxamine dihydrochloride $(10 \mu \mathrm{M}), \mathrm{BML}-111(10 \mu \mathrm{M}), \mathrm{L} 3223(10$ $\mu \mathrm{M})$, Enalapril maleate $(10 \mu \mathrm{M})$, Emricasam $(10 \mu \mathrm{M})$, CWHM12 $(1 \mu \mathrm{M})$, C8 $(10 \mu \mathrm{M})$, Cyclo (RGDyK) $(0.04 \mu \mathrm{M})$, Cyclo (RGDfK) $(0.1$ $\mu \mathrm{M})$, SB273005 $(10 \mu \mathrm{M})$, Cilengitide $(1 \mu \mathrm{M})$, DC9711 (10 $\mu \mathrm{M})$. Cells were then lysed, and the ECM was fixed and stained using a total protein dye or specific antibodies. Graph shows the effect of each small molecule at the dose indicated (highest dose before effect on cell viability was observed) on the total ECM. DMSO control $(0.1 \%)$ is also shown. Data are represented as fold change from the DMSO control and show the mean with $95 \%$ CIs from 2 independent experiments with 4 replicate wells per condition. ${ }^{*} p<0.05$, ${ }^{* *} p<0.01,{ }^{* * *} p<0.001,{ }^{* * * *} p<0.0001$; Analysis of variance, fitting groups and allowing for the different experiments and comparison of each inhibitor to the DMSO-matched control using Dunnett's test. ns, not significant; ECM, extracellular matrix.
Against a large backdrop of in vivo multi-organ models of fibrosis, there are very few translatable in vitro models with a disease-relevant read-out, and in this study we have described a primary kidney epithelial: fibroblast coculture cell system that quantitates the disease-relevant ECM signal. There is a lack of studies examining the effect of knocking down integrins in vitro and measurement of a fibrotically relevant phenotype. Osteoblastic osteosarcoma MG63 cells deficient in aV were unable to elaborate a fibronectin matrix and lost fibrillin 1 expression [38]. Biochemical and functional characteristics of the $\alpha \mathrm{V}$ integrins described in the literature centre around the binding of fibronectin, vitronectin and LAP-TGF- $\beta 1 / 3$ and subsequent cellular signalling events [23,39]. We could speculate that the $\alpha \mathrm{V}$ integrins are having a role at multiple points along the activation process in the renal contact co-culture ECM accumulation assay. First, the initial epithelial: fibroblast cell contact could be mediated via $\alpha \mathrm{V}$ integrin-ligand binding events. This initial cell contact is crucial for the spontaneous ECM response as it is not observed in the mono-cultures of either cell type or when they are in co-culture but separated via a transwell system. Second, $\alpha V$ integrins could initiate the release of active TGF- $\beta 1 / 3$ from pre-formed latent TGF $\beta$ that is stored either in the early ECM or cell-associated. There is accumulating evidence that there is extensive crosstalk between integrins and TGF- $\beta$ signalling and this interaction is reciprocal [40]. TGF- $\beta$ directly controls integrin expression, and conversely, the $\alpha \mathrm{V}$ integrins directly regulate TGF- $\beta$ activation [32].

Third, fibronectin: cell adhesion, potentially mediated via $\alpha \mathrm{V}$, could propagate further cell activation and signalling to stimulate the extracellular accumulation of the interstitial collagens, which have been shown in this study to deposit at the latter time points. In our study, we noted that fibronectin deposition was one of the first matrix proteins to be deposited in the co-culture. Fibronectin is a high affinity ligand of $\alpha \mathrm{V}$ integrins [41], and 
fibronectin polymerization has been shown to be a critical regulator of ECM organization and stability, which subsequently controls cell-matrix adhesion and signalling events [42]. It is interesting that the collagens colocalised spatially with fibronectin, supporting the "scaffolding" role for fibronectin in the formation of the fibrillar collagen ECM. Fourth, $a V$ integrins have been described to bind some pro-fibrotic growth factors and growth factor receptors, such as $\alpha \mathrm{V} \beta 3$ binding of vascular endothelial growth factor receptor 2 [43] and $\alpha \mathrm{V} \beta 6$ binding of connective tissue growth factor (CTGF) [44], both of which are linked to fibrogenesis. Renal fibroblasts proliferate and differentiate into myofibroblasts in response to many pro-fibrotic growth factors, and the renal co-culture ECM signal is potently inhibitable with the multi-kinase growth factor inhibitor, nintedanib.

In summary, we have demonstrated that the $\alpha \mathrm{V}$ family of integrins can functionally regulate the accumulation of ECM in a renal co-culture in vitro model of fibrosis, which was central to our hypothesis. It has been shown that the direct interaction between epithelial cells and fibroblasts is imperative in mediating this matrix accumulation and requires the $\alpha \mathrm{V}$ integrins. The pan$\alpha \mathrm{V}$ inhibitors were amongst the most potent inhibitors at knocking down the ECM accumulation after 7 days of co-culture and this was the case for both neutralising antibodies and small molecule inhibitors. This cell model has been validated with the clinically relevant anti-fibrotic, nintedanib and inhibitors of TGF- $\beta$, the most widely described fibrotic mediator. The anti-pan $\alpha \mathrm{V}$ blocking antibody was by far the most potent antibody in inhibiting the ECM response, outperforming the anti-TGF- $\beta$ antibody. As targeting of TGF- $\beta$ as an antifibrotic therapeutic is unlikely to be successful given all the other homeostatic and immunological properties of this central regulator, the $\alpha \mathrm{V}$ integrins provide a viable alternative strategy for therapeutic intervention. This is unlikely to be achieved through the use of a pan $\alpha \mathrm{V}$ blocker due to potential toxicology concerns; however, a more specific approach could be adopted to target the individual family members that have also shown efficacy in this study. Given that the integrin $\beta 1$ is highly expressed in both epithelial cells and fibroblasts, the expression is maintained in the co-culture and the small molecule $\alpha \mathrm{V} \beta 1$ inhibitor, $\mathrm{C} 8$, potently inhibits the ECM signal, it is tempting to speculate that blocking the $\alpha \mathrm{V} \beta 1$ axis could lead to beneficial anti-fibrotic clinical effects without the potential damaging side effects of global $\alpha \mathrm{V}$ inhibition. Very significant inhibition was also observed with both $\alpha \mathrm{V} \beta 6$ inhibitors (both ab77906 blocking antibody and DC9711 small molecule) and although $\beta 6$ expression is restricted to epithelial cells, it may also be a relevant target for therapeutic intervention. It will be interesting to observe the progress of the 2 current clinical trials in IPF targeting the $\alpha \mathrm{V} \beta 6$ integrin to determine if blocking this specific axis can modify disease progression without an adverse event burden. Important future considerations for integrin targeting anti-fibrotic therapies will include the identification of likely combinatorial regimens and development of efficacy biomarkers to track performance. We have also characterized a very translatable robust model of fibrosis that could be more widely applied in the hunt for future anti-fibrotic therapeutics.

The data presented here support the hypothesis that the $\alpha \mathrm{V}$ integrins play a central role in the ECM accumulation response mediated by the interaction of fibroblasts and epithelial cells and support their targeting in fibrotic disease.

\section{Acknowledgements}

This study was funded by UCB Pharma. We thank Halima Aliyu for preparing plates for immunofluorescence.

\section{Statement of Ethics}

All human primary cells used in this study were sourced commercially from companies following appropriate consent and ethics, complying with the UK human tissue authority guidelines.

\section{Disclosure Statement}

H.B., O.Q., P.H., S.L., G.H., T.J. and B.T. were employees of UCB Pharma and hold UCB Pharma shares and/or stock options. This work was funded by UCB Pharma.

\section{Author Contributions}

H.B. and O.Q.: designed the study, performed experiments and were involved in the analysis and interpretation of the data. B.T., G.H., and T.J.: were involved in the design, analysis and interpretation of the data. H.B., O.Q., T.J., and B.T.: wrote the manuscript with input from the other authors. P.H. and S.L.: carried out the gene expression and analyses of expression. 


\section{References}

1 Taal MW. Chronic kidney disease: towards a risk-based approach. Clin Med (Lond). 2016 Dec;16(Suppl 6):s117-20.

2 Tampe D, Zeisberg M. Potential approaches to reverse or repair renal fibrosis. Nat Rev Nephrol. 2014 Apr;10(4):226-37.

3 Meng XM, Nikolic-Paterson DJ, Lan HY. TGF- $\beta$ : the master regulator of fibrosis. Nat Rev Nephrol. 2016 Jun;12(6):325-38.

4 Wei Y, Kim TJ, Peng DH, Duan D, Gibbons DL, Yamauchi M, et al. Fibroblast-specific inhibition of TGF- $\beta 1$ signaling attenuates lung and tumor fibrosis. J Clin Invest. 2017 Oct; 127(10):3675-88.

5 Moll S, Ebeling M, Weibel F, Farina A, Araujo Del Rosario A, Hoflack JC, et al. Epithelial cells as active player in fibrosis: findings from an in vitro model. PLoS One. 2013;8(2): e56575.

6 Holdsworth G, Bon H, Bergin M, Qureshi O, Paveley R, Atkinson J, et al. Publisher Correction: quantitative and organisational changes in mature extracellular matrix revealed through high-content imaging of total protein fluorescently stained in situ. Sci Rep. 2017 Dec;7(1):18111.

7 Qureshi OS, Bon H, Twomey B, Holdsworth G, Ford K, Bergin M, et al. An immunofluorescence assay for extracellular matrix components highlights the role of epithelial cells in producing a stable, fibrillar extracellular matrix. Biol Open. 2017 Oct;6(10): 1423-33.

8 Fine LG, Norman JT, Ong A. Cell-cell crosstalk in the pathogenesis of renal interstitial fibrosis. Kidney Int Suppl. 1995 Jun;49:S48-50.

$9 \mathrm{Xu}$ J, Mora AL, LaVoy J, Brigham KL, Rojas $\mathrm{M}$. Increased bleomycin-induced lung injury in mice deficient in the transcription factor T-bet. Am J Physiol Lung Cell Mol Physiol. 2006 Oct;291(4):L658-67.

10 Henderson NC, Arnold TD, Katamura Y, Giacomini MM, Rodriguez JD, McCarty JH, et al. Targeting of av integrin identifies a core molecular pathway that regulates fibrosis in several organs. Nat Med. 2013 Dec;19(12): 1617-24.

11 Bagnato GL, Irrera N, Pizzino G, Santoro D, Roberts WN, Bagnato G, et al. Dual $\alpha v \beta 3$ and av $\beta 5$ blockade attenuates fibrotic and vascular alterations in a murine model of systemic sclerosis. Clin Sci (Lond). 2018 Jan;132(2):231-42.

12 Marek I, Lichtneger T, Cordasic N, Hilgers KF, Volkert G, Fahlbusch F, et al. Alpha8 Integrin (Itga8) Signalling Attenuates Chronic Renal Interstitial Fibrosis by Reducing Fibroblast Activation, Not by Interfering with Regulation of Cell Turnover. PLoS One. 2016 Mar;11(3):e0150471.

13 Zheng G, Zhang J, Zhao H, Wang H, Pang M, Qiao X, et al. a3 Integrin of Cell-Cell Contact Mediates Kidney Fibrosis by Integrin-Linked Kinase in Proximal Tubular E-Cadherin Deficient Mice. Am J Pathol. 2016 Jul;186(7): 1847-60.
14 Raab-Westphal S, Marshall JF, Goodman SL. Integrins as Therapeutic Targets: successes and Cancers. Cancers (Basel). 2017 Aug;9(9):

15 Hall ER, Bibby LI, Slack RJ. Characterisation of a novel, high affinity and selective $\alpha v \beta 6$ integrin RGD-mimetic radioligand. Biochem Pharmacol. 2016 Oct;117:88-96.

16 Nanthakumar CB, Hatley RJ, Lemma S, Gauldie J, Marshall RP, Macdonald SJ. Dissecting fibrosis: therapeutic insights from the small-molecule toolbox. Nat Rev Drug Discov. 2015 Oct;14(10):693-720.

17 Greiling D, Clark RA. Fibronectin provides a conduit for fibroblast transmigration from collagenous stroma into fibrin clot provisional matrix. J Cell Sci. 1997 Apr;110(Pt 7):86170.

18 Wollin L, Wex E, Pautsch A, Schnapp G, Hostettler KE, Stowasser S, et al. Mode of action of nintedanib in the treatment of idiopathic pulmonary fibrosis. Eur Respir J. 2015 May;45(5):1434-45.

19 Fukihara J, Kondoh Y. Nintedanib (OFEV) in the treatment of idiopathic pulmonary fibrosis. Expert Rev Respir Med. 2016 Dec;10(12): 1247-54.

20 Akhurst RJ, Hata A. Targeting the TGF $\beta$ signalling pathway in disease. Nat Rev Drug Discov. 2012 Oct;11(10):790-811.

21 Rice LM, Padilla CM, McLaughlin SR, Mathes A, Ziemek J, Goummih S, et al. Fresolimumab treatment decreases biomarkers and improves clinical symptoms in systemic sclerosis patients. J Clin Invest. 2015 Jul;125(7): 2795-807.

22 Trachtman H, Fervenza FC, Gipson DS, Heering $\mathrm{P}$, Jayne DR, Peters $\mathrm{H}$, et al. A phase 1, single-dose study of fresolimum$\mathrm{ab}$, an anti-TGF- $\beta$ antibody, in treatmentresistant primary focal segmental glomerulosclerosis. Kidney Int. 2011 Jun;79(11): 1236-43.

23 Wright DB, Meurs H, Dekkers BG. Integrins: therapeutic targets in airway hyperresponsiveness and remodelling? Trends Pharmacol Sci. 2014 Nov;35(11):567-74.

24 Dasch JR, Pace DR, Waegell W, Inenaga D, Ellingsworth L. Monoclonal antibodies recognizing transforming growth factor-beta. Bioactivity neutralization and transforming growth factor beta 2 affinity purification. J Immunol. 1989 Mar;142(5):1536-41.

25 Mitjans F, Sander D, Adán J, Sutter A, Martinez JM, Jäggle CS, et al. An anti-alpha v-integrin antibody that blocks integrin function inhibits the development of a human melanoma in nude mice. J Cell Sci. 1995 Aug; 108(Pt 8):2825-38.

26 Reardon DA, Cheresh D. Cilengitide: a prototypic integrin inhibitor for the treatment of glioblastoma and other malignancies. Genes Cancer. 2011 Dec;2(12):1159-65.

27 Miller WH, Alberts DP, Bhatnagar PK, Bondinell WE, Callahan JF, Calvo RR, et al. Dis- covery of orally active nonpeptide vitronectin receptor antagonists based on a 2-benzazepine Gly-Asp mimetic. J Med Chem. 2000 Jan; 43(1):22-6

28 Gabison EE, Huet E, Baudouin C, Menashi S. Direct epithelial-stromal interaction in corneal wound healing: role of EMMPRIN/ CD147 in MMPs induction and beyond. Prog Retin Eye Res. 2009 Jan;28(1):19-33.

29 Sakai N, Tager AM. Fibrosis of two: epithelial cell-fibroblast interactions in pulmonary fibrosis. Biochim Biophys Acta. 2013 Jul; 1832(7):911-21.

30 Nugraha B, Mohr MA, Ponti A, Emmert MY, Weibel F, Hoerstrup SP, et al. Monitoring and manipulating cellular crosstalk during kidney fibrosis inside a $3 \mathrm{D}$ in vitro co-culture. Sci Rep. 2017 Nov;7(1):14490.

31 Choi K, Lee K, Ryu SW, Im M, Kook KH, Choi C. Pirfenidone inhibits transforming growth factor- $\beta 1$-induced fibrogenesis by blocking nuclear translocation of Smads in human retinal pigment epithelial cell line ARPE-19. Mol Vis. 2012;18:1010-20.

32 Dong X, Zhao B, Iacob RE, Zhu J, Koksal AC, $\mathrm{Lu} C$, et al. Force interacts with macromolecular structure in activation of TGF- $\beta$. Nature. 2017 Feb;542(7639):55-9.

33 Wipff PJ, Rifkin DB, Meister JJ, Hinz B. Myofibroblast contraction activates latent TGFbetal from the extracellular matrix. J Cell Biol. 2007 Dec;179(6):1311-23.

34 Horan GS, Wood S, Ona V, Li DJ, Lukashev $\mathrm{ME}$, Weinreb $\mathrm{PH}$, et al. Partial inhibition of integrin alpha(v)beta6 prevents pulmonary fibrosis without exacerbating inflammation. Am J Respir Crit Care Med. 2008 Jan;177(1): 56-65.

35 Munger JS, Huang X, Kawakatsu H, Griffiths MJ, Dalton SL, Wu J, et al. The integrin alpha v beta 6 binds and activates latent TGF beta 1: a mechanism for regulating pulmonary inflammation and fibrosis. Cell. 1999 Feb;96(3): 319-28.

36 Liu S, Xu SW, Blumbach K, Eastwood M, Denton CP, Eckes B, et al. Expression of integrin beta1 by fibroblasts is required for tissue repair in vivo. J Cell Sci. 2010 Nov;123(Pt 21): 3674-82.

37 Reed NI, Jo H, Chen C, Tsujino K, Arnold TD, DeGrado WF, et al. The av $\beta 1$ integrin plays a critical in vivo role in tissue fibrosis. Sci Transl Med. 2015 May;7(288):288ra79.

38 Boregowda RK, Krovic BM, Ritty TM. Selective integrin subunit reduction disrupts fibronectin extracellular matrix deposition and fibrillin 1 gene expression. Mol Cell Biochem. 2012 Oct;369(1-2):205-16.

39 Goodman SL, Picard M. Integrins as therapeutic targets. Trends Pharmacol Sci. 2012 Jul;33(7):405-12.

40 Margadant C, Sonnenberg A. Integrin-TGFbeta crosstalk in fibrosis, cancer and wound healing. EMBO Rep. 2010 Feb;11(2):97105. 
41 Plow EF, Haas TA, Zhang L, Loftus J, Smith JW. Ligand binding to integrins. J Biol Chem. 2000 Jul;275(29):21785-8.

42 Sottile J, Hocking DC. Fibronectin polymerization regulates the composition and stability of extracellular matrix fibrils and cell- matrix adhesions. Mol Biol Cell. 2002 Oct; 13(10):3546-59.

43 Somanath PR, Malinin NL, Byzova TV. Cooperation between integrin alphavbeta 3 and VEGFR2 in angiogenesis. Angiogenesis. 2009;12(2):177-85.
44 Pi L, Robinson PM, Jorgensen $\mathrm{M}$, Oh $\mathrm{SH}$, Brown AR, Weinreb PH, et al. Connective tissue growth factor and integrin $a v \beta 6$ : a new pair of regulators critical for ductular reaction and biliary fibrosis in mice. Hepatology. 2015 Feb;61(2):678-91. 